\title{
NON-UNIQUE FLOWS IN MACROSCOPIC FIRST-ORDER INTERSECTION MODELS
}

\author{
Ruben Corthout $^{1 *}$, Gunnar Flötteröd ${ }^{2}$, Francesco Viti ${ }^{1}$, Chris M.J. Tampère ${ }^{1}$
}

May 20, 2011

Report TRANSP-OR 110520

Transport and Mobility Laboratory

Ecole Polytechique Fédérale de Lausanne

transp-or.epf l.ch

${ }^{1}$ Department of Mechanical Engineering, CIB/Traffic \& Infrastructure, Katholieke Universiteit Leuven,

Celestijnenlaan 300A, PO Box 2422, 3001 Leuven, Belgium

*E: ruben.corthout@ cib.kuleuven.be; T: +32 (0)16 3296 14; F: +32 (0)16 322987

${ }^{2}$ Transport and Mobility Laboratory (TRANSP-OR), Ecole Polytechnique Fédérale de Lausanne (EPFL),

Station 18, 1015 Lausanne, Switzerland 


\begin{abstract}
Currently, most intersection models embedded in macroscopic Dynamic Network Loading (DNL) models are not well suited for urban and regional applications. This is so because socalled internal intersection supply constraints, bounding flows due to crossing and merging conflicts inherent to the intersection itself, are missing. This paper discusses the problems that arise upon introducing such constraints, which result firstly from a lack of empirical knowledge on driver behavior at general intersections under varying conditions and the incompatibility of existing theories that describe this behavior with macroscopic DNL. A generic framework for the distribution of (internal) supply is adopted, which is based on the definition of priority parameters that describe the strength of each flow in the competition for a particular supply. Secondly, using this representation, it is shown that intersection models even under realistic behavioral assumptions and in simple configurations (i.e. without internal supply constraints) - can produce non-unique flow patterns under identical boundary conditions. This solution non-uniqueness is thoroughly discussed and conceptual approaches on how it can be dealt with in the model are provided. Also the spatial modeling point of view is considered - as opposed to the more traditional point-like modeling. It is revealed that the undesirable model properties are not solved - but rather enhanced - when diverting from a point-like to a spatial modeling approach. Therefore, we see more merit in continuing the point-like approach for the future development of sophisticated intersection models. Necessary research steps along these lines are formulated.
\end{abstract}

Keywords: first-order macroscopic intersection model, Dynamic Network Loading, internal intersection supply constraints, solution non-uniqueness

\title{
1 Introduction
}

In first-order macroscopic dynamic network loading (DNL) models, the link model provides the demand of incoming links and the supply of outgoing links as boundary conditions to the intersection model. These intersection (or node) models are typically point-like, i.e. without physical dimensions, combining all internal and external constraints into one strongly coupled set of equations.

The function of the intersection model is then two- or threefold. Firstly, it seeks for a consistent solution in terms of flows transferred over the intersection, accounting for all demand and supply constraints of the adjacent links. Secondly, it imposes additional constraints due to limited supply of conflict points on the intersection itself; these are called internal intersection supply constraints. The latter typically do not apply to highway junctions but can be decisive at regional and urban intersections. These two functions determine the flows over the intersection and (if applicable) the congestion dynamics in the adjacent links. Finally, imposing additional delay at the intersection itself, e.g. based on delay formulas such as those of Akcelik \& Troutbeck (1991) and Webster (1958) constitutes a third function in models that do not explicitly capture stochastic queue formations in under-saturated conditions (e.g. Durlin \& Henn, 2005; Yperman et al., 2007). In this paper, the focus is on the first two functions, which determine the flows. In the following Section 1.1, the state-of-theart regarding these two functions is discussed. Section 1.2 is devoted to an elaboration on the intention, scope and contributions of this paper. 


\subsection{State-of-the-art on first-order DNL intersection models}

The first function of the intersection model, combining demand and supply constraints into a consistent solution, is severely complicated by the fact that (the distribution of) the supplies and the resulting flows are not independent. As discussed in Tampère et al. (2011), this dependency should be captured by a supply constraint interaction rule (SCIR). Multiple plausible definitions of this rule are conceivable, but it should realistically represent the aggregate driver behavior at a congested intersection. Furthermore, Tampère et al. (2011) list a set of requirements with which first-order macroscopic intersection models in DNL should comply:

1. General applicability to any number of incoming links and outgoing links

2. Non-negativity of flows

3. Conservation of vehicles

4. Compliance with demand constraints and (internal) supply constraints

5. Flow maximization from the users' perspective

6. Conservation of turning fractions (CTF)

7. Compliance with the invariance principle of Lebacque \& Khoshyaran (2005)

The first four requirements are rather straightforward and well-known in the literature. The latter three deserve a little more explanation. For this, we introduce the following notation: incoming (upstream) links of the considered intersection are indexed by $i=1 \ldots I$ and outgoing (downstream) links are indexed by $j=1 \ldots J$. The demand (supply) of link $i(j)$ is denoted by $S_{i}\left(R_{j}\right)$. The total demand $S_{i}$ consists of partial demands $S_{i j}$ in the various outgoing directions, defined by the turning fractions $f_{i j}$ so that $S_{i j}=f_{i j} S_{i}$. The flow sent by link $i$ to link $j$ is written as $q_{i j}$, and the total outflow of $i$ is $q_{i}=\sum_{j} q_{i j}$.

- Flow maximization from the users' perspective:

Each flow should be actively constrained by either demand or (internal) supply. Behaviorally, this reflects the assumption of drivers trying to advance whenever possible. This corresponds to individual flow maximization, not global flow maximization. The latter is an unrealistic modeling assumption: why would (selfish) drivers be inclined to behave cooperatively?

- Conservation of turning fractions (CTF):

This requirement states that the outflow composition of a link $i$ (in terms of partial flows $q_{i j}$ in the various downstream directions) must be identical to its demand composition (in terms of $S_{i j}$ ). Consequently, all outflows of $i$ are coupled through the turning fractions that are obtained from the demand composition:

$$
f_{i j}=\frac{S_{i j}}{S_{i}}=\frac{q_{i j}}{q_{i}}
$$

CTF implies First-In-First-Out (FIFO) at the intersection level (Daganzo, 1995). Due to CTF, the intersection model's solution is unambiguously defined by the incoming flows $q_{i}$; the partial flows are then derivable as $q_{i j}=$ $f_{i j} q_{i}$. 
- Compliance with the invariance principle of Lebacque \& Khoshyaran (2005): The invariance principle ensures the compatibility of the intersection model with the link traffic flow dynamics and can be formulated as follows. If the intersection model produces $q_{i}<S_{i}$, then $i$ enters a congested regime. As a consequence of traffic flow dynamics, $S_{i}$ increases after some infinitesimally small time increment to the link's capacity $C_{i}$. Any intersection model that predicts a different outcome for $q_{i}$ because of this change from $S_{i}$ to $C_{i}$ contradicts its own initial solution and thus violates the invariance principle. The solution of the intersection model should therefore be invariant to replacing $S_{i}$ by $C_{i}$ if $q_{i}$ is supply constrained $\left(q_{i}<S_{i}\right)$. Analogously, if $q_{j}<R_{j}$ the solution should be invariant to an increase of $R_{j}$ to $C_{j}$.

The review of state-of-the-art intersection models in Tampère et al. (2011) shows that most existing models fail to comply with some or several of the above requirements and as such do not fulfill the first function of intersection models properly. Apart from the two intersection models introduced in Tampère et al. (2011) - one for unsignalized and one for signalized intersections -, adequate intersection models that correctly combine demand and supply constraints according to the above requirements are presented by Flötteröd \& Nagel (2005), Flötteröd (2008), Gentile (2010), Gibb (2011) and Flötteröd \& Rohde (forthcoming). However, these models still do not incorporate internal supply constraints (except due to traffic control), except for Flötteröd \& Rohde (forthcoming).

In fact, internal intersection supply constraints, which constitute the second function of intersection models, are rarely considered in state-of-the-art models. There are several causes for this. Firstly, it is a difficult task to formulate these internal intersection supply constraints. Quite some studies exist that estimate the throughput of minor streams yielding to higher prioritized flows - e.g. Chapters 8 and 9 in Gartner et al. (2000), Brilon \& Wu (2001) and Brilon \& Miltner (2005). However, these formulations assume an uncongested traffic state downstream. Secondly, the internal supply constraints are dependent on the resulting flows. This also further complicates the correct fulfillment of the first function.

Intersection models in the traditional point-like form that do include internal intersection supply constraints are presented in Ngoduy et al. (2005), Yperman et al. (2007), Chevallier \& Leclercq (2007) and Raadsen et al. (2010). Spatial intersection models accounting for internal supply constraints are introduced by Buisson et al. (1995) and Chen et al. (2008). However, none of these models comply with all of the above listed requirements. The model specification of Flötteröd \& Rohde (forthcoming) - as mentioned before - does comply, but a uniquely converging solution algorithm in the presence of internal supply constraints is presented only for the special case where the incoming links can be ranked such that the resulting flows from these links are independent from those of lower ranked links. Indeed, the impossibility to design a solution algorithm that is proven to converge to a unique solution leads to the (possibly seminal) investigation of solution uniqueness presented in that article. Motivated by the identification of a simple (three-legged) configuration that already yields non-unique flows, the authors provide a heuristic algorithm with guaranteed convergence towards a compromise solution.

\subsection{Scope of the paper}

On top of the seven requirements of Tampère et al. (2011), additional specifications and considerations are needed to develop adequate intersection models that account for internal 
conflicts. Including internal intersection supply constraints lifts the model complexity to a much higher level. A compromise between realism on the one hand and complexity on the other is probably unavoidable. Currently, the commonly adopted balance is to completely ignore the internal supply constraints. In many regional or urban DNL applications, however, this is unacceptable since these constraints are (largely) responsible for the traffic problems in such networks. The additional modeling of these constraints, the resulting methodological difficulties and the accompanying research questions are formulated and discussed in this paper.

The first problem is which behavioral assumptions are to be made regarding intersection conflicts and how this can be modeled by internal intersection supply constraints in a DNL intersection model. Section 2 starts by identifying three types of driver behavior: strict and limited compliance to priority rules and turn-taking. A classification of intersection conflicts under varying saturation levels into these three types of behavior is suggested. These findings are partially derived from available empirical studies. Since not all types of conflicts have been (sufficiently) documented, additional assumptions are necessary. As such, until validated empirically, our classification is susceptible to discussion.

Section 2.2 introduces the translation of these behavioral considerations into theories to be embedded in the DNL intersection model, and the difficulties that arise therewith. In Section 2.3, a generic framework that extends existing models such as Daganzo (1995) and Flötteröd \& Rohde (forthcoming) is presented. The distribution of external and internal supply constraints is described in terms of priority parameters (or functions). It is shown how these can be adapted to model a broad spectrum of driver behavior.

Secondly, this article further develops the findings of Flötteröd \& Rohde (forthcoming) on the solution non-uniqueness of intersection flows. This discussion benefits from the generic framework in terms of priority parameters defined in Section 2.3. In Section 3, we reason from the traditional point-like modeling approach. We identify multiple-valued priority ratios among incoming flows $q_{i}$ that are tied in several (internal) supply constraints as a general source of non-uniqueness (Sections 3.1 and 3.2). It is shown that even for intersection models that do not consider internal supply constraints, uniqueness of the solution is not trivially guaranteed. This applies, e.g., to the models of Flötteröd \& Nagel (2005) and Gentile (2010). Yet, in the state-of-the-art, uniqueness is usually implicitly assumed.

Comparison can be made to the commonly adopted dynamic traffic assignment (DTA) framework, which is also known to have multiple solutions in quite realistic cases (Daganzo, 1998). Therefore, this paper is conceptually similar to Carey (1992), who shows that the FirstIn-First-Out (FIFO) behavior of traffic can lead to non-unique solutions in DTA and also proposes how to practically deal with this problem.

In intersection models, we see two ways to deal with the observed non-uniqueness, namely stochastically and deterministically. In stochastic DNL models (e.g. Sumalee et al., 2011 and Osorio et al., forthcoming), non-unique intersection flows could be permitted. Just as a stochastic approach to the DTA problem yields a unique solution in distributional terms (e.g., Flötteröd \& al., forthcoming), a stochastic DNL could replace non-unique intersection flows by a unique distribution. In deterministic DNL modeling, a transformation of the non-unique solutions into one prevailing flow pattern is needed, which seems less straightforward. Depending on the application, one might favor the stochastic or the deterministic approach. For both approaches, empirical studies with the aim of describing the non-uniqueness in reality would be highly valuable (see Section 3.3). Even if such studies were to disprove the existence of non-uniqueness in reality, still the issue has to be dealt with in the models. In 
Section 3.4, some pragmatic approaches to deal with the non-uniqueness in deterministic models are suggested.

Finally, Section 4 discusses spatial intersection modeling, following the suggestion of Flötteröd \& Rohde (forthcoming) that modeling intersections with physical dimensions might alleviate the non-uniqueness encountered in point-like models. However, it is shown that also spatial models can produce different flow patterns under identical boundary conditions, and that the result is inherently determined based on the history of flows. Moreover, some unrealistic and undesirable model behavior is identified. We therefore see better potential in research that aims at further developing traditional point-like models rather than abandoning them in favor of spatial models.

\section{From driver behavior to (internal) supply constraints}

Apart from the quite straightforward constraints imposed by signals or other types of control, internal intersection supply constraints arise from conflicts between crossing and merging flows. Intersection supply constraints encompass:

- Traffic controls (traffic lights, ramp metering)

- Crossing conflicts

$O$ at (un)signalized intersections, between movements originating from different incoming links, heading towards different outgoing links

○ with non-motorized traffic (pedestrians, cyclists)

- Merging conflicts

$\circ$ between flows merging into an outgoing link

$\circ$ between flows entering a roundabout, merging with flows already on the roundabout

It should be noted that conflicts between flows merging into the same outgoing link - listed as an internal constraint here - are typically considered as external constraints in the form of the outgoing link's supply. In fact, they can be considered partially internal and partially external: on the one hand, they can be dominated by congestion spilling back from the outgoing link. On the other hand, drivers usually evaluate crossing and merging conflicts simultaneously before traversing the intersection.

In this paper, only motorized traffic is considered. Also, conflicts due to traffic controls are not discussed since these are either - in case of non-adaptive control - quite straightforward to deal with or - in case of adaptive control - to the best of our knowledge, not included in stateof-the-art DNL models.

\subsection{Driver behavior in crossing and merging conflicts}

In the following, a distinction is made between three types of driver behavior in solving intersection conflicts, namely strict compliance to priority rules, limited compliance to priority rules, and turn-taking. In reality, different driver behavior can be observed depending on traffic load, intersection type and geometry, and personal and cultural differences.

Strict compliance to priority rules covers cases where an ordering of the movements is imposed, rendering priority to some movements over others. In theory, according to the traffic 
rules, this behavior should apply to merging and crossing conflicts at general intersections under all circumstances. In reality, this behavior can be observed for crossing and merging conflicts at low traffic volumes (under-saturated).

As traffic volumes increase, priority rules are less strictly obeyed due to politeness from prioritized and forcing from non-prioritized drivers. This limited compliance behavior commonly arises as the minor movements are in nearly- to over-saturated conditions, while in general the major flows are not supply constrained - they may however incur some additional delay. This kind of behavior is documented empirically for roundabout merging in Troutbeck \& Kako (1999) and for crossing and merging flows in Brilon \& Miltner (2005).

In over-saturated conditions, typically some sort of turn-taking behavior occurs - at least for merging conflicts - which can be regarded as the alternating use of the available supply by all competing movements. Hereby, the 'turns' or opportunities that are available for each movement are determined by the movements' outflow capacities, possibly reduced by other conflicts (crossing, control). As such, 'pure' turn-taking behavior overrides the imposed priority rules in that each movement obtains a priority that is determined by its physical capacity. Several factors may influence this behavior, making it difficult to recognize and define in a general way. Empirical studies of merging behavior in over-saturated conditions at general intersections are lacking. Cassidy \& Ahn (2005) find that congested freeway merging occurs in a fixed, site-dependent ratio, independently of the available downstream supply. This is further confirmed in Bar-Gera \& Ahn (2010), who show that this fixed ratio is well approximated by the ratio of the number of lanes of the incoming links of the merge. From a smaller data set, Ni \& Leonard (2005) conclude that merging follows the ratio of the capacities (which is of course very similar to number of lanes). Finally, All-Way-StopControlled (AWSC) intersections actually prescribe turn-taking for both merging and crossing conflicts. Although for AWSC intersections this type of behavior is thus in compliance with the priority rules, the behavior at AWSC conflicts is categorized as turn-taking and the concepts of limited and strict compliance are preserved for situations as described above.

Naturally, the aggregate driver behavior to be captured by macroscopic intersection models may be a mixture of different types of behavior. In Figure 1, a suggestion is provided on how aggregate driver behavior at various intersection conflicts could be classified. This classification is certainly subject to numerous factors, which are often unknown or immeasurable, and it is thus not indisputable.

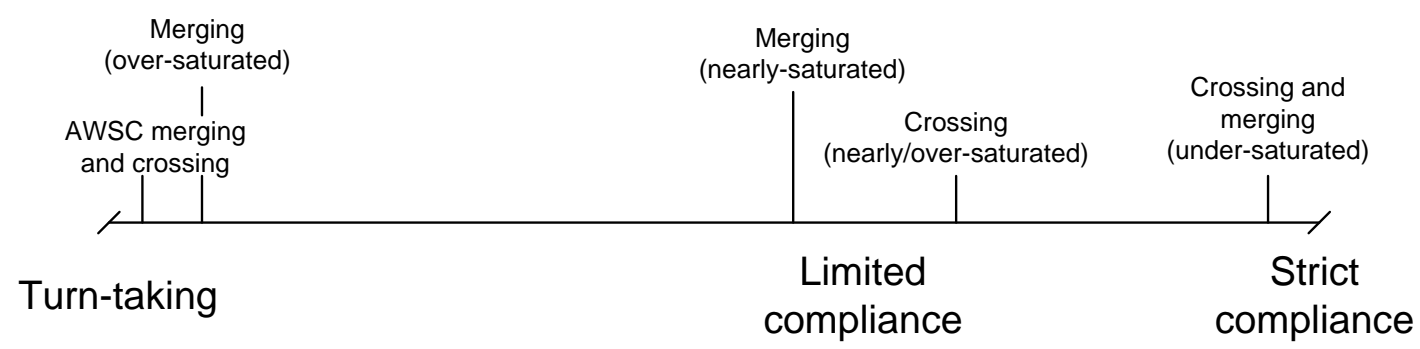

Figure 1: Driver behavior in intersection conflicts

\subsection{Existing theories describing observed behavior}

Before constructing a DNL intersection model, theories are needed that describe how to model the observed behavior discussed in the previous section. Turn-taking at merging conflicts is generally modeled by some sort of distribution rule (see Tampère et al. (2011) for 
a thorough discussion). All further discussion on modeling turn-taking is reserved for the next section. Regarding the other two types of behavior, strict and limited compliance, a few theories exist to determine the restriction on minor movements from yielding to higher prioritized movements in crossing and merging conflicts. The best-known is gap acceptance theory (see Chapters 8 and 9 in Gartner et al., 2000), which found a more recent counterpart in conflict theory (Brilon \& Wu, 2001). Most often these theories assume strict compliance with priority rules, but modifications exist for limited compliance (Troutbeck \& Kako, 1999; Brilon \& Miltner, 2005). Although some existing DNL intersection models embed gap acceptance theory to model internal conflicts (Yperman et al., 2007; Ngoduy et al., 2005; Chevallier \& Leclercq, 2007; Flötteröd \& Rohde, forthcoming), neither gap acceptance theory nor conflict theory was initially designed for use in a DNL context. The incompatibility of these theories with DNL stems from two aspects.

Firstly, both theories are designed for free flowing conditions of the major stream. Gap acceptance theory in particular loses some validity in nearly-saturated and definitely in oversaturated conditions. Conflict theory is, at least theoretically, extendable to over-saturated conditions; but this has not been validated empirically. Bridging the different types of driver behavior in DNL intersection models by embedding, supplementing and adapting these existing theories - or developing new ones - therefore constitutes important future research.

A second problem stems from the first one. As is discussed in Tampère et al. (2011), multiple demand and/or supply constraints interact with each other, causing flows from different incoming links to be mutually dependent. This must be captured in a supply constraint interaction rule (SCIR); see Section 1.1. This possibility of mutual dependency is not considered in gap acceptance and conflict theory, as the major flows are considered known (i.e. equal to the demand). In a DNL intersection model, however, the major flows are also subject to (internal) supply constraints and therefore not known beforehand. A few existing models (Yperman et al., 2007; Ngoduy et al., 2005) that implement internal supply constraints derived from gap acceptance theory solve this by making the internal supply constraints that limit the minor flows dependent on the demands of the major streams instead of the actual major flows. As explained in Section 1, this leads to a violation of the invariance principle if the major streams are not demand constrained. Moreover, this simplification may cause an overestimation of the constraint imposed on the minor flows (Flötteröd \& Rohde, forthcoming).

\subsection{Modeling (internal) supply constraints in DNL intersection models}

As discussed in Section 2.1, different driver behavior can be observed under varying conditions - to be roughly subdivided in strict compliance, limited compliance, and turntaking. In the following, priority parameters $\alpha$ are introduced through which these different types of driver behavior are modeled.

An intuitive way of representing the solution space is used (as in Daganzo, 1995). Figure 2 exemplifies this graphical representation for a two-legged merge. Firstly, the demand constraints $S_{1}$ and $S_{2}$ set upper bounds on the flow from their respective incoming link.

Secondly, the external supply constraint function $R_{3}\left(q_{1}, q_{2}\right) \leq 0$ further reduces the solution space. The circumflex is introduced to make a distinction between the supply constraint function and the actual supply $R_{3}$ that link 3 can receive. In the state-of-the-art, this supply constraint function is typically written in the form

$$
R_{3}\left(q_{1}, q_{2}\right)=q_{1}+q_{2}-R_{3} \leq 0 \text {. }
$$


Daganzo (1995) introduces priority parameters $\alpha_{1}$ and $\alpha_{2}$ that represent the strength of links 1 and 2 in the competition for the restrictive supply $R_{3}$. If both $q_{i}$ are supply constrained by $R_{3}$ - which is denoted by $q_{i} \mapsto R_{3}$ - the supply distribution based on $\alpha_{1}$ and $\alpha_{2}$ renders the following solution:

$$
q_{1}=\frac{\alpha_{1} R_{3}}{\alpha_{1}+\alpha_{2}} ; q_{2}=\frac{\alpha_{2} R_{3}}{\alpha_{1}+\alpha_{2}} \quad \text { if } q_{1}, q_{2} \mapsto R_{3}
$$

In this case, the priority ratio $\frac{\alpha_{2}}{\alpha_{1}}$ and the binding supply constraint $\left(R_{3}\left(q_{1}, q_{2}\right)=0\right)$ together dictate the unique solution point $\mathrm{A}$ in Figure 2:

$$
\frac{q_{2}}{q_{1}}=\frac{\alpha_{2}}{\alpha_{1}} \quad \text { if } q_{1}, q_{2} \mapsto R_{3}
$$

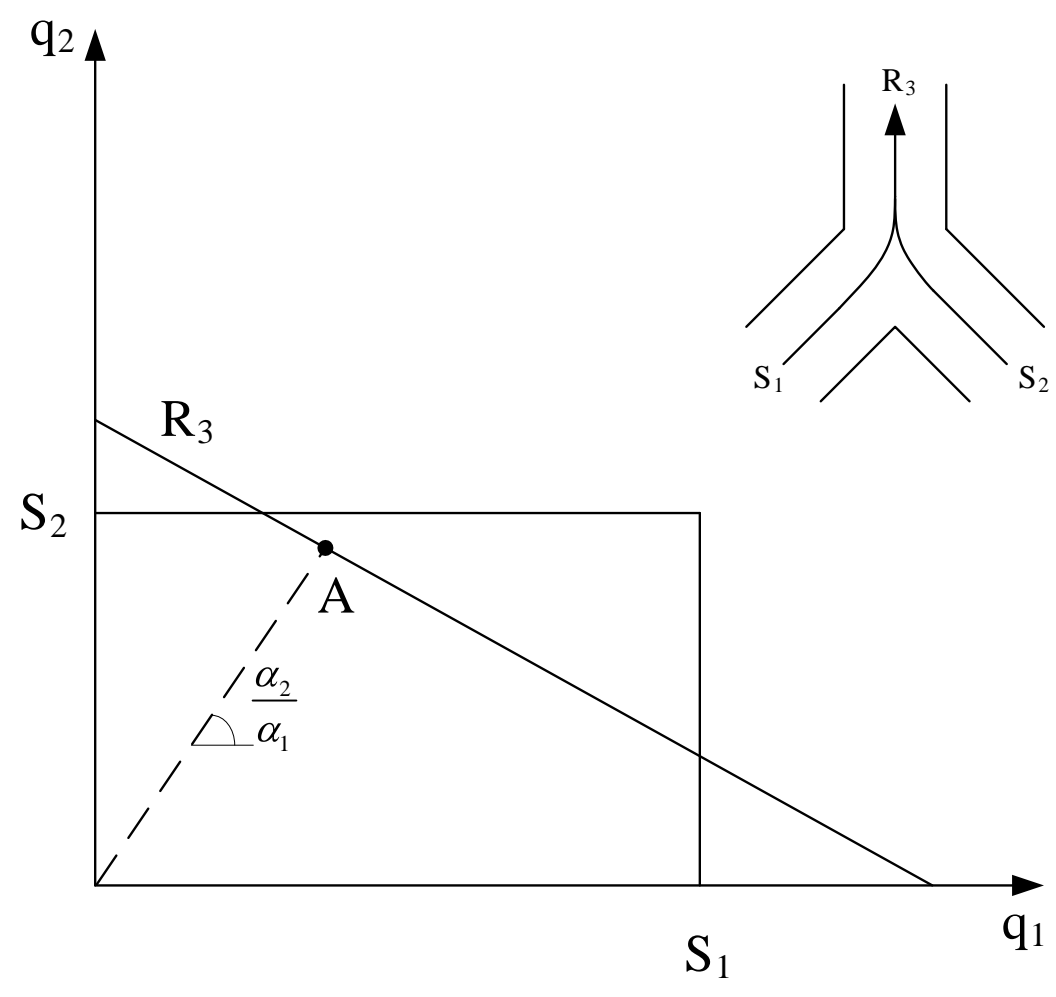

Figure 2: Supply constrained solution in merge model (Daganzo, 1995)

Most existing general intersection models only implement the supply constraints of the outgoing links' supplies, and can thus be regarded as direct generalizations of Daganzo's merge model in that they extend relationship (2) into:

$$
R_{j}\left(q_{1}, \ldots, q_{I}\right)=f_{1 j} q_{1}+\ldots+f_{I j} q_{I}-R_{j} \leq 0 \quad \forall j
$$

Now, a framework is defined that unifies general intersection models that comply with the 7 requirements listed in Section 1.1 and that encompass a SCIR (see Tampère et al., 2011), 
controlling the interaction of supply constraints and their distribution on the basis of priority parameters. First, this is explained in terms of external supply constraints, afterwards it is extended to internal constraints.

Finite, positive priority parameters $\alpha_{i j}$ are considered so that the strength of $i$ in the competition for supply $R_{j}$ is given by $f_{i j} \alpha_{i j}$. As such, $\alpha_{i j}$ can be regarded as the maximal strength (if $f_{i j}=1$ ). It is indeed logical that the turning fractions $f_{i j}$ play an explicit role; the competitive strength of $i$ for $R_{j}$ is naturally zero if $f_{i j}=0$. For a particular $i$ ' constrained by $R_{j}$ ( $\left.R_{j}\left(q_{1}, \ldots, q_{I}\right)=0\right)$, the supply distribution (3) then generalizes to:

$$
q_{i^{\prime} j}=\frac{\alpha_{i^{\prime} j} f_{i^{\prime} j} R_{j}}{\sum_{i} \alpha_{i j} f_{i j}} \Rightarrow q_{i^{\prime}}=\frac{\alpha_{i^{\prime} j} R_{j}}{\sum_{i} \alpha_{i j} f_{i j}} \quad \text { if } q_{i^{\prime}}, q_{i} \mapsto R_{j}
$$

Typically, it is assumed that one most restrictive constraint can be identified that determines the flow from a link $i$. If the share of $R_{j}$ is too much to consume for some incoming links $\bar{i}-$ due to more restrictive demand or other supply constraints - the remaining supply $R_{j}-\sum_{i} q_{i j}$ is divided among the remaining $i$ analogous to (6) - for more details see Tampère et al. (2011). Also, we presume that vehicles only enter the intersection if they have the opportunity to exit via their destination link, so that external supplies $R_{j}$ do not directly influence flows to other outgoing directions. In other words, only a link $i$ that wishes to send flow to a link $j$ (i.e. $S_{i j}>0$ ) participates in the competition for $R_{j}$. Finally, for any combination of incoming links $i$ and $i$ ' that are constrained by the same supply $R_{j}$, the combination of (6) with the CTF requirement renders the following solution (analogous to (4)):

$$
\frac{q_{i}}{q_{i^{\prime}}}=\frac{\alpha_{i j}}{\alpha_{i^{\prime} j}} \quad \forall i, i^{\prime} \mid q_{i}, q_{i^{\prime}} \mapsto R_{j}
$$

The main difference between models lies in how the priority parameters $\alpha_{i j}$ are defined. In the following it is explained how the priority parameters can be used to model different driver behavior in external and internal conflicts. Hereby it should be noted that while the priority parameters are typically set as constant values, they might as well be functions of the turning fractions, capacities or flows (as in the models of Gibb, 2011 and Flötteröd \& Rohde, forthcoming). Consequently, transitions in driver behavior, e.g. depending on the saturation level (see Figure 1), may be accounted for by priority functions rather than constants. For simplicity's sake, we regard the priority parameters as constants in the remainder of the paper, unless stated otherwise.

Regarding external supply constraints, most existing intersection models assume a singlevalued priority parameter $\alpha_{i}$ for a link $i$, which is then used in the competition for all $R_{j} \mid S_{i j}>$ 0 . If an external supply constraint is active, i.e. the merging conflict is over-saturated, this is typically solved through turn-taking behavior (see Section 2.1). Turn-taking behavior is modeled by basing $\alpha_{i}$ on the number of lanes or capacities of the incoming links, e.g. $\alpha_{i}=C_{i}$ (as for example in Tampère et al., 2011). Empirical studies on freeway merges (e.g. Bar-Gera \& Ahn, 2010) support this common modeling practice of assuming fixed priority parameters for any value of $R_{j}$ to represent turn-taking behavior. 
Analogous as for external supplies in outgoing links $j$, internal supplies in intersection conflicts $k$ put constraints on the flows $q_{i}$. Thus, internal supply constraint functions $N_{k}\left(q_{1}, \ldots, q_{I}\right)$ can be considered that bound all subjected flows $\left(S_{i k}>0\right)$. This could for example stem from the conflict area shared by crossing or merging movements. The internal supply constraint functions $N_{k}$ are more difficult to define than the $R_{j}$. They are to be derived from gap acceptance or conflict (or any new) theory, as discussed in Section 2.2. Exactly defining these functions $N_{k}$ is beyond the scope of this paper. However, we can state, analogous to (7):

$$
\frac{q_{i}}{q_{i^{\prime}}}=\frac{\alpha_{i k}}{\alpha_{i^{\prime} k}} \quad \forall i, i^{\prime} \mid q_{i}, q_{i^{\prime}} \mapsto N_{k}
$$

Very similar to Figure 2, the solution space for a simple intersection with one crossing conflict (represented by $\left.N_{3}\right)$ is depicted in Figure 3. $N_{3}\left(q_{1}, q_{2}\right) \leq 0$ limits $q_{1}$ and $q_{2}$ that compete for the shared internal supply. Say that for this intersection, the priority rules prescribe that $q_{2}$ has priority over $q_{1}$. Strict compliance to this priority rule could then be modeled by setting $\alpha_{1}=0^{1}$ and $\alpha_{2}=1$. In consequence, $q_{2}$ fully consumes its maximal share (hence $q_{2}=S_{2}$ ), leaving the remaining internal supply for $q_{1}$, yielding solution $A$. Limited compliance could be modeled by less extreme values, e.g. $\alpha_{1}^{*}>0$ and $\alpha_{2}^{*}<1$ yielding $A^{*}$ in Figure 3. Recall that if turn-taking behavior applies to the crossing conflict (as in AWSC intersections), the $\alpha_{i}$ are based on the capacities $C_{i}$.

\footnotetext{
${ }^{1}$ At this point, we should add that at most one $\alpha_{i j}$ (or $\alpha_{i k}$ ) can be defined zero per $j(k)$. In priority-controlled intersections, always a ranking exists between any two movements that are in conflict. In cases of turn-taking behavior, zero priorities do not make sense altogether.
} 


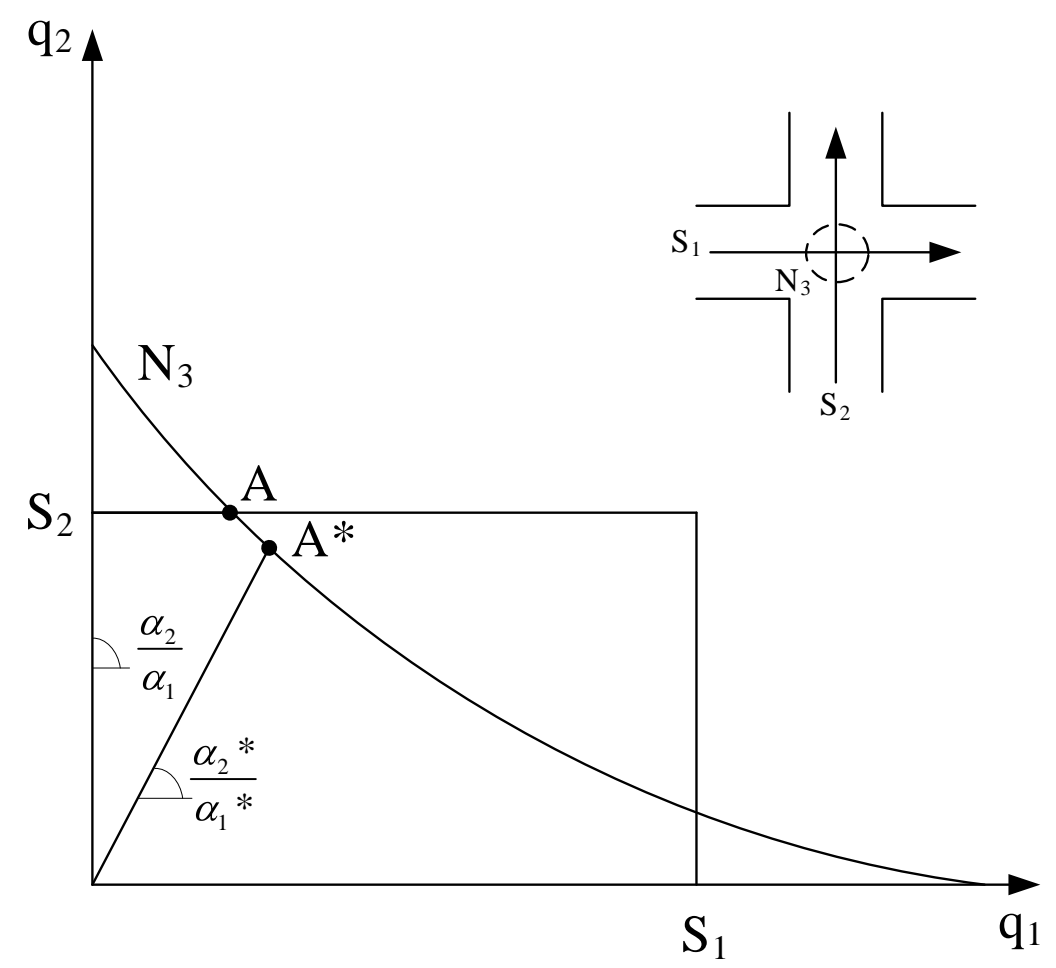

Figure 3: Internal supply constrained solution

Note that the internal supply constraint function $N_{3}$ may be non-linear, for instance because the consumption rate of the internal supply may be different for $q_{1}$ and $q_{2}$, or because of joint constraints, which derive from the fact that drivers evaluate crossing and merging conflicts on general intersections simultaneously (unless some storage capacity for crossing vehicles is present on the intersection). Consider for example the situation in Figure 4, where a minor flow from the westbound link has to yield to bi-directional traffic of the major street. A vehicle from the minor street will only cross if no prioritized vehicle is approaching from either side.

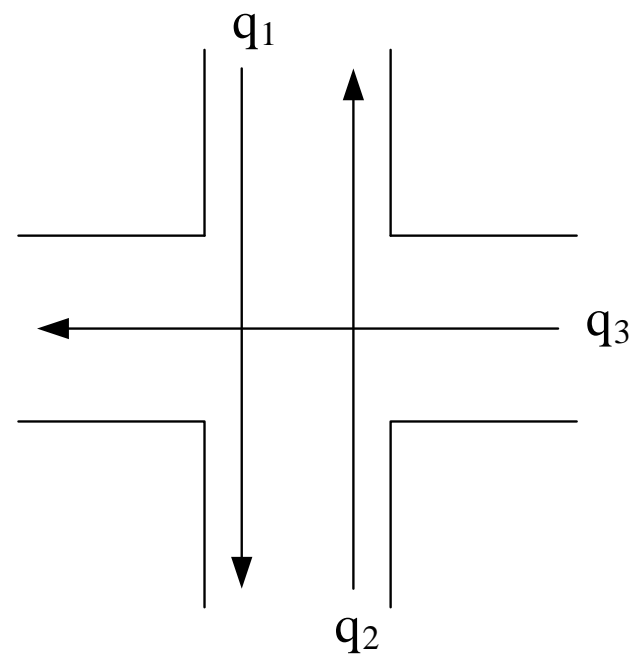

Figure 4: Minor movement yielding to bi-directional traffic 
This suggests to combine these two internal conflicts into one joint internal supply constraint function $N\left(q_{1}, q_{2}, q_{3}\right) \leq 0$. Regardless, the solution (space) of the intersection model can still be represented as in Figure 3. The definition of the priority parameters may, however, be more complicated in case of joint constraints.

In conclusion, this section explains in general terms how priority parameters can be used to model different types of driver behavior. The depth and complexity of this discussion is limited to the level necessary to introduce the concepts of modeling internal supply constraints due to merging and crossing conflicts and to elaborate on the solution nonuniqueness observed therein, as discussed in the next sections.

\section{Solution non-uniqueness in point-like intersection models}

In this section, intersection models in the traditional point-like form are discussed, whereas spatial models are reserved for Section 4. Firstly, the problem of solution non-uniqueness is introduced by means of two simple examples in Section 3.1. Then, this non-uniqueness is further analyzed in Section 3.2. Section 3.3 raises the question whether the problem of nonuniqueness, which arises in the intersection model from realistic behavioral assumptions, would in fact be identifiable in reality. Finally, some pragmatic approaches to deal with nonuniqueness in deterministic intersection models are given in Section 3.4.

\subsection{Explanatory examples}

In the literature, solution uniqueness of the intersection model is often implicitly assumed. However, the following example shows that even for simple cases, which only consider external supply constraints, uniqueness of the solution is not self-evident.

\subsubsection{Example 1 (only external supply constraints)}

A simple 2x2-intersection is considered (Figure 5), where the intersection model assumes different priority parameters $\alpha_{i j}$ for an incoming link $(i=1,2)$ per outgoing link $(j=3,4)$. This means that the incoming links 1 and 2 have a different priority (maximal strength) in the competition for $R_{3}$ and $R_{4}$; this could for instance be based on the number of turning lanes. This corresponds to the intersection models in Gentile (2010) and Flötteröd \& Nagel (2005). The solution space defined by the demand and external supply constraints is depicted in Figure 5.

Due to the CTF requirement, both inflows $q_{i}$ are mutually tied in both supply constraints. As such, the solution is fully determined by the total inflows $q_{i}$ from which the partial flows $q_{i j}$ derive (see Section 1.1). If $R_{j}\left(q_{1}, q_{2}\right)=0$ (see (5)) and both $q_{i} \mapsto R_{j}$, the priority parameters $\alpha_{i j}$ determine the solution according to (7) (or (6)). 


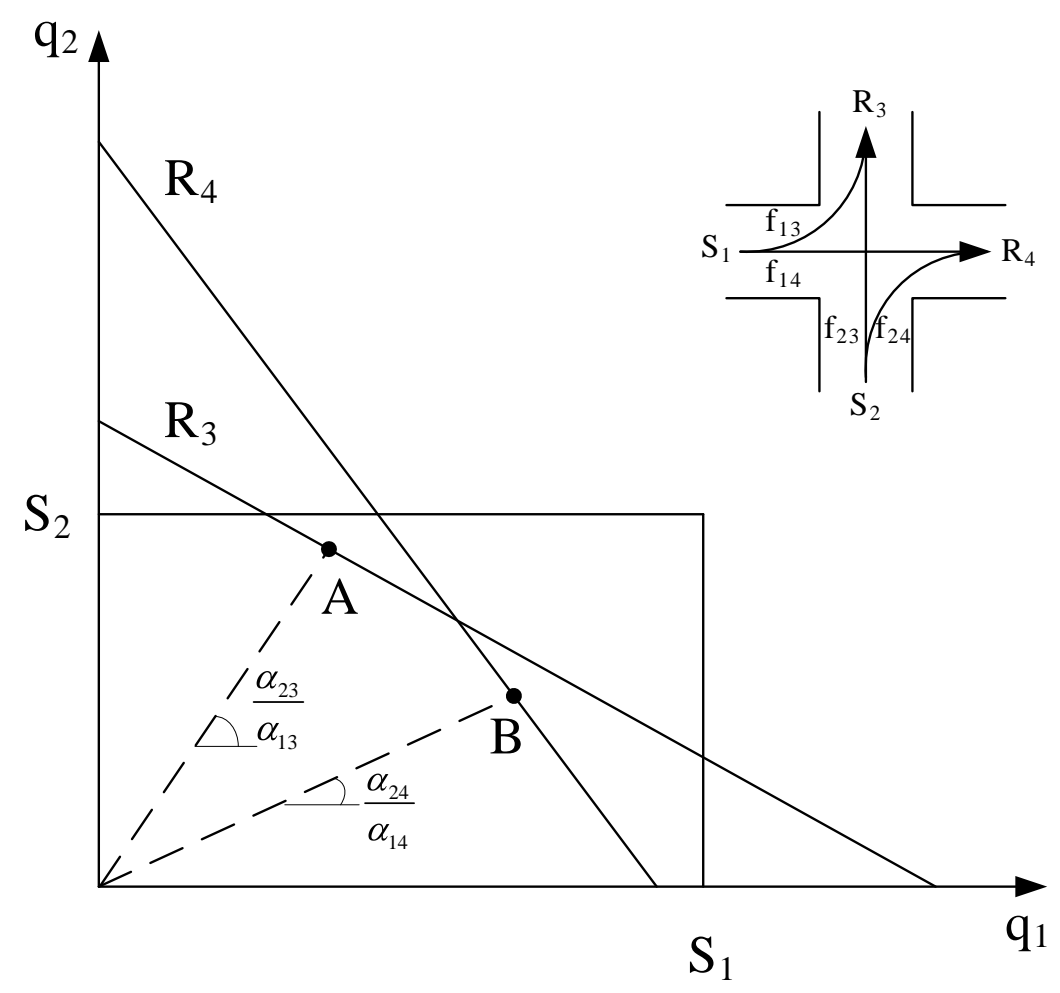

Figure 5: Solution non-uniqueness example 1

In this example, there are two possible solutions ( $A$ and $B$ ) satisfying all constraints and the distribution rule defined by the $\alpha_{i j}$, depending on which supply constraint is considered restrictive. This shows that even in simple models without internal intersection supply constraints, solution non-uniqueness can be an issue. To our knowledge, this problem has never been addressed in the literature; Flötteröd \& Rohde (forthcoming) only discuss internal supply constraints. Although most existing models assume single-valued priority parameters $\alpha_{i}$ - which would solve the problem - multiple-valued parameters $\alpha_{i j}$ are considered in some models (e.g. Flötteröd \& Nagel (2005), Gentile (2010), Adamo et al., 1999; Ni et al., 2006; Taale, 2008). Hereby we should note that Flötteröd \& Nagel (2005) ultimately resort to the computation of one "representative" priority parameter. Also, in the latter three models the use of separate $\alpha_{i j}$ does not induce solution non-uniqueness as these models ignore CTF. This is due to the fact that as flows from a link $i$ into various directions $j$ are detached, the individual $q_{i j}$ are no longer tied in multiple supply constraints. Each $q_{i j}$ is in this case subject to only one supply constraint and can thus be determined via its individual $\alpha_{i j}$. However, even without CTF, uniqueness is not guaranteed once internal supply constraints are added; see Section 3.2.

\subsubsection{Example 2 (with internal supply constraints)}

The following example is a $2 \times 4$ intersection, which can be interpreted as a standard 4-leg intersection where only two inflows are considered. Other flows can be regarded as negligible or at least not decisive for the solution.

In the upper right part of Figure 6, two crossing conflicts can be identified. The priority rules for such conflicts typically state that the left turning movement has to yield to the straight 
movement coming from the opposite link, i.e. $\alpha_{23}=\alpha_{14}=1$ and $\alpha_{13}=\alpha_{24}=0$ (see Section 2.3). Considering only the demand constraints and these internal supply constraints (in conjunction with CTF), a solution space as in Figure 6 results.

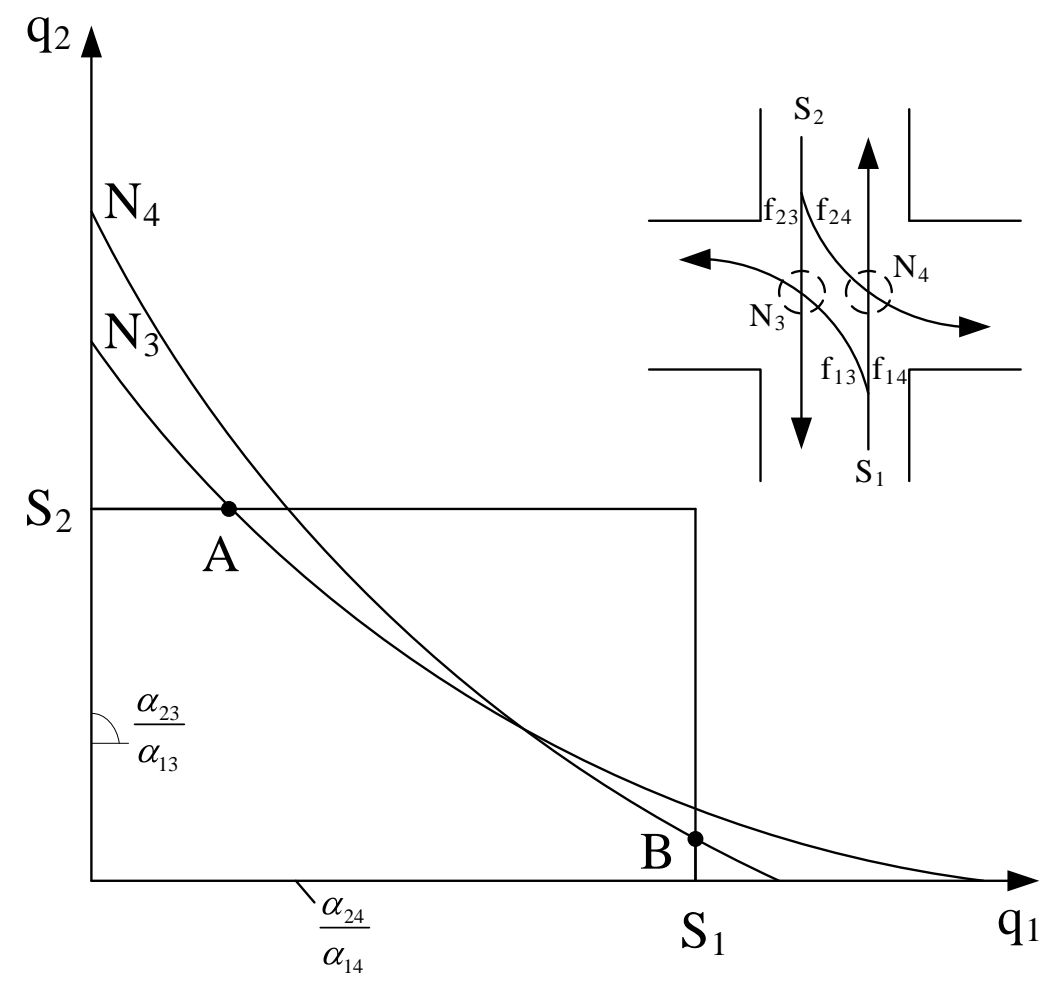

Figure 6: Solution non-uniqueness example 2

Again there are two possible solutions $\left(A\right.$ and $B$ ), depending on whether the distribution of $N_{3}$ or $N_{4}$ is followed. The cause of the non-uniqueness is the same as in the previous example, namely the inflows $q_{i}$ being faced with different priority ratios for different supply constraints. Note that even if limited compliance is assumed, there would still be two different priority ratios and hence two solutions. Thus, in intersection models that include internal supply constraints, solution non-uniqueness arises from realistic behavioral assumptions.

\subsection{General analysis of solution non-uniqueness}

In the following, we assume intersection models to comply with the 7 requirements listed in Section 1.1 and to follow the rules regarding supply distribution as described in Section 2.3.

The previous section illustrates the occurrence of solution non-uniqueness in simple examples. We identify the general source of this non-uniqueness in the intersection model as the fact that inflows $q_{i}$, coupled through multiple (internal) supply constraints (and usually $\mathrm{CTF}$ ), are faced with multiple, ambiguous priority ratios. For this, let $F_{i}$ be the set of (internal) supplies for which $i$ competes, i.e.:

$$
F_{i}=\left\{j, k \mid S_{i j}, S_{i k}>0\right\}
$$


Now, there are two ways in which flows $q_{i}$ can be mutually dependent through multiple supply constraints:

1. Two (or more) inflows $q_{i_{1}}$ and $q_{i_{2}}$ are tied in two (or more) supply constraints, i.e. $\#\left(F_{i_{1}} \cap F_{i_{2}}\right) \geq 2$.

2. Three (or more) inflows $q_{i_{1}}, q_{i_{2}}$ and $q_{i_{3}}$ are mutually dependent in some "circular way", so that each (internal) supply constraint binds two different inflows in a sequence, returning from the last $i$ to the first, forming a circle in which all inflows are mutually dependent, i.e.: $F_{i_{1}} \cap F_{i_{2}}=\left\{j_{1}\right\}, F_{i_{2}} \cap F_{i_{3}}=\left\{j_{2}\right\}, F_{i_{3}} \cap F_{i_{1}}=\left\{j_{3}\right\}$. Or in other words: $S_{i_{1} j_{1}}>0, S_{i_{2} j_{1}}>0, S_{i_{2} j_{2}}>0, S_{i_{3} j_{2}}>0, S_{i_{3} j_{3}}>0, S_{i_{1} j_{3}}>0$ with all other $S_{i j}=0$ (any $j$ could of course be replaced by a $k$ ).

Naturally, combinations of 1 and 2 can exist. Option 1 is the more likely of the two to appear in an intersection model. Note that this option implicitly assumes CTF, since otherwise the $q_{i j}$ are detached and thus two inflows $q_{i}$ cannot be reasonably tied in the same two (internal) supply constraints. Further in this section, an example of the second, less obvious option is provided, which shows that solution non-uniqueness can also exist in non-CTF models.

Now, a sufficient and necessary condition for solution uniqueness of the intersection flows is that each priority parameter $\alpha_{i j}$ and $\alpha_{i k}$ of inflows $q_{i}$ that are mutually dependent in supply constraints in $j$ and $k$ can be written as the product of a single-valued $\alpha_{i}$ for each $i$ and a factor $\beta$ for each (internal) supply constraint in $j$ or $k$ :

$$
\exists \alpha_{i}, \beta_{j}, \beta_{k}: \alpha_{i j}=\alpha_{i} \beta_{j} \& \alpha_{i k}=\alpha_{i} \beta_{k} \quad \forall i \forall j, k \in F_{i}
$$

with

$$
\begin{array}{ll}
\alpha_{i} \geq 0 & \forall i \\
\beta_{j}>0 & \forall j \\
\beta_{k}>0 & \forall k
\end{array}
$$

The factors $\beta_{j}$ and $\beta_{k}$ are scalable, as is the set of $\alpha_{i}$ 's in its entirety. As such, a sufficient condition for solution uniqueness can be derived from the above by setting all $\beta_{j}$ and $\beta_{k}$ to one, thus defining single-valued priority parameters $\alpha_{i}$ for each link $i$ to be applied in the distribution of all (internal) supplies.

Proof for condition (10) is added in appendix. For flows $q_{i}$ that are mutually dependent in the first manner described above, (10) reads as 'the priority ratios are identical for all (internal) supply constraints in which these $q_{i}$ are both involved', thus ruling out ambiguity and nonuniqueness.

To illustrate the second manner for mutual dependency, let us regard the example in Figure 7. An intersection is considered with three incoming flows $q_{i}(i=1 \ldots 3)$ and three internal supply constraint functions $N_{k}(k=4 \ldots 6)$ each encompassing two $q_{i}$. As such, the constraint functions are two-dimensional planes (they only form a triangle in the graph because the same function is assumed for each constraint - which seems to make most sense). Now, the set of $\alpha_{i}$ 's in (10) is presented as a vector $\overrightarrow{\alpha_{i}}$ of which the direction is unique, but not the norm - as 
the set of $\alpha_{i}$ 's is scalable. If all $\beta_{k}=1, \overrightarrow{\alpha_{i}}$ represents the resulting single-valued priority parameters. Its unique direction ensures that a unique priority ratio exists between each two flows $q_{i}$; for a particular $k$, this ratio is formed by the two coordinates of $\overrightarrow{\alpha_{i}}$ of the two $i$ that compete in that $k$. In other words, the priority ratio in $k$ is the projection of $\overrightarrow{\alpha_{i}}$ in the 2-D plane of $i \mid S_{i k}>0$. The unique solution is determined by this vector $\overrightarrow{\alpha_{i}}$, i.e. whichever supply constraint it 'hits' first. The solution is not depicted to avoid overloading the graph; also demand constraints are not considered. Now, scaling the priority parameters $\alpha_{i}$ with factors $\beta_{k} \neq 1$ leads to multiple-valued priority parameters $\alpha_{i k}$, that still meet (10). In Figure 7, this is represented by three priority ratios $\frac{\alpha_{i k}}{\alpha_{i^{\prime} k}}=\frac{\alpha_{i} \beta_{k}}{\alpha_{i^{\prime}} \beta_{k}}$. As these are parallel to the projections of $\overrightarrow{\alpha_{i}}$, the priority ratios remain unchanged and thus the same unique solution results.

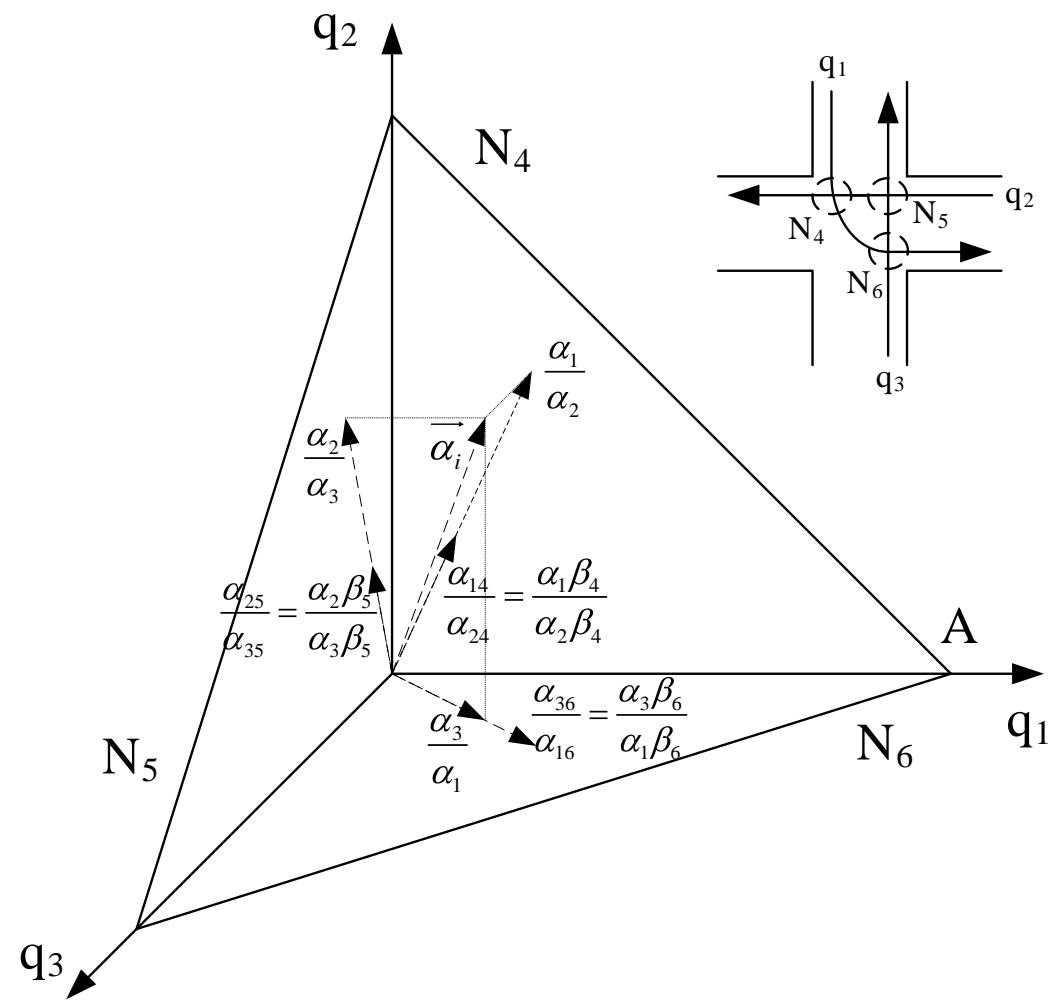

Figure 7: Graphical illustration of requirement (10)

Reconsidering the above example, it becomes clear that the CTF requirement - which is assumed throughout the paper - is not always part of the problem of solution non-uniqueness. Assume the following priority parameters in the same example, which correspond to "priority to the right': $\alpha_{14}=1 ; \alpha_{24}=0 ; \alpha_{25}=1 ; \alpha_{35}=0 ; \alpha_{36}=1 ; \alpha_{16}=0$. As such, multiple, ambiguous priority ratios produce three solutions $A, B$ and $C$ (Figure 8). Obviously, CTF is not a factor here, since all flows $q_{i}$ are unidirectional. 


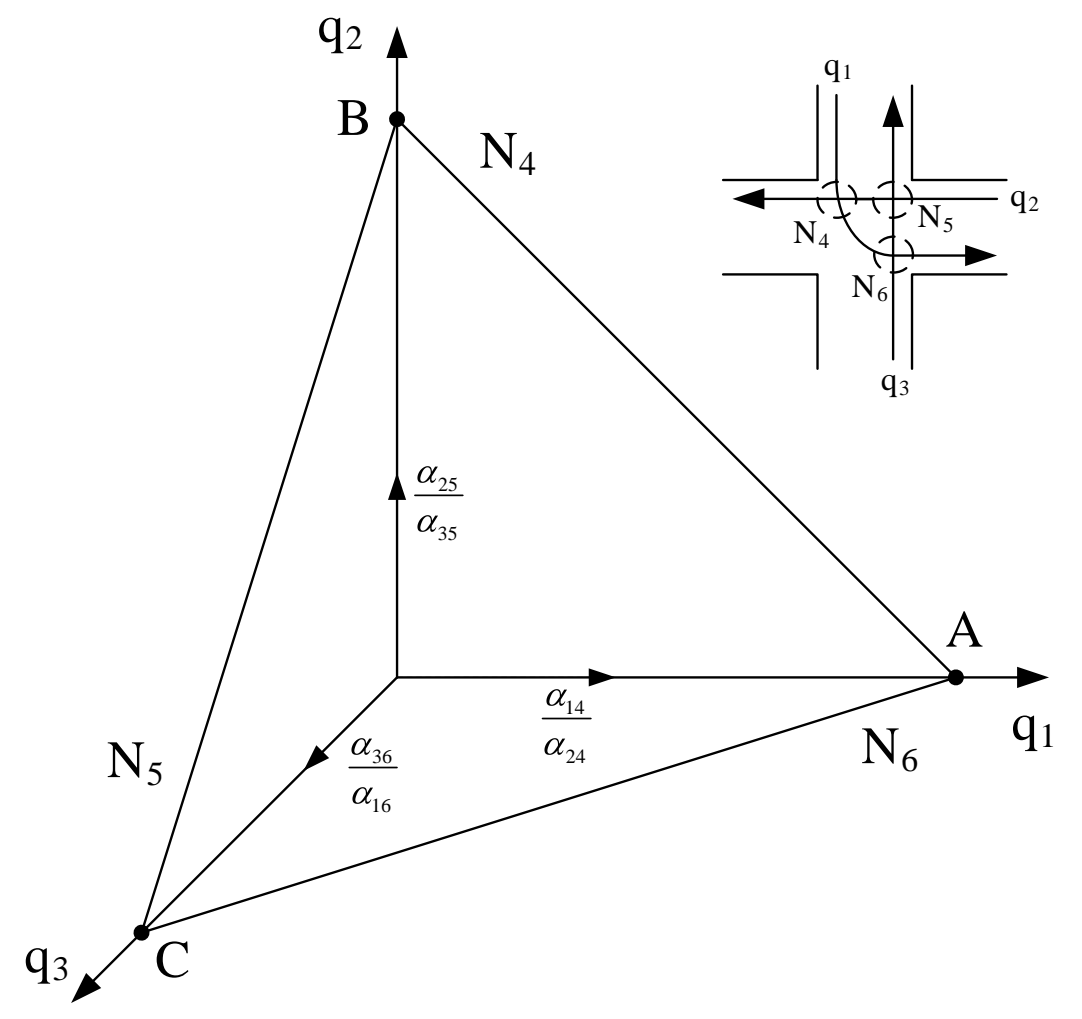

Figure 8: Example of non-uniqueness when CTF is not a factor

Of course, depending on the boundary conditions - the demand, supplies and turning fractions - ambiguous priority ratios may or may not induce multiple solutions in a specific case. For instance, one supply constraint may dominate and define one unique solution. The nonuniqueness will appear only under quite specific circumstances, which troubles empirical validation.

\subsection{Non-uniqueness in the model and reality}

In the previous section, we identified the condition (10), to which the priority parameters $\alpha_{i j}$ and $\alpha_{i k}$ must comply so that the solution of the intersection model is unique. For models that only consider external supply constraints, this condition does not form an insuperable problem. In such models, choosing single-valued priority parameters $\alpha_{i}$ (i.e. meeting condition (10) with all $\beta_{j}=1$ ) is a commonly adopted and accepted modeling assumption. For internal intersection constraints, however, multi-valued priority parameters $\alpha_{i k}$ result from realistic behavioral assumptions. As discussed in Section 2.1, different driver behavior (and thus different priorities) can be observed depending on the type of conflict and the saturation level. Furthermore, even one type of behavior - as in Section 3.1.2 where full compliance is assumed for both conflicts - may translate into ambiguous priority ratios and thus non-uniqueness. Condition (10) is therefore counterintuitive and probably an unrealistic modeling assumption if internal supply constraints are involved.

This means that whether or not multiple solutions exist in reality, the possibility of solution non-uniqueness in the model has to be acknowledged and dealt with. Preferably, however, this should be supported by empirical research. The empirical studies on intersection flows conducted in the past do not provide sufficient support since their focus is typically on various 
aspects of gap acceptance behavior (e.g. headway distributions) and not on validating intersection models in DNL (except for simple merges; see e.g. Bar-Gera \& Ahn, 2010 and Ni $\&$ Leonard, 2005). Flow non-uniqueness has never been reported in empirical data, but it has also never been looked for. Since non-uniqueness only appears in the model under specific circumstances, where multiple supply constraints can dominate under identical boundary conditions, identifying these circumstances constitutes a considerable practical difficulty. Additionally, the need for a large amount of detailed data - not only traffic counts, but also video observation - renders this empirical research labor- and cost-intensive.

\subsection{Pragmatic approaches to deal with non-uniqueness in deterministic intersection models}

Regardless of whether empirical research is to confirm or disprove the existence of multiple solutions in reality, the non-uniqueness has to be dealt with in the intersection model. In stochastic models, a unique probability distribution, encompassing the multiple solutions, could be composed. For deterministic intersection models, we suggest in the following some pragmatic approaches that produce unique solutions. Further developing these into actual models requires also extending the other theoretical concepts discussed in this paper, i.e. explicitly defining internal supply constraint functions and priority parameters. Moreover, the development of efficient algorithms constitutes a considerable challenge.

First of all, it is stressed that the following two approaches to try to remedy the nonuniqueness should be avoided:

- disregarding CTF

- extending the requirement of individual flow maximization to global flow maximization

Not only do these suffer from unrealistic modeling assumptions; uniqueness is not fully guaranteed in either approach. For non-CTF cases, this is demonstrated by the example in Section 3.2. Regarding global flow maximization, if the total flow $\sum_{i} q_{i}$ is the same for multiple solutions, a global maximization is also subject to non-uniqueness.

A model definition in which solution uniqueness is ensured can be obtained by imposing (10). This is in line with the modeling assumptions in many state-of-the-art intersection models that consider only external supply constraints. As stated before, this assumption lacks realism for most types of intersection conflicts. Only if the expected driver behavior is limited to turntaking behavior, as in AWSC intersections, reverting to this approach seems natural, e.g. with $\alpha_{i}=C_{i}$.

It may be desirable to at first allow ambiguous priorities in the model definition, and then to alleviate the non-uniqueness by some kind of pre- or post-processing. In the following, we distinguish two types of approaches: (a) pre-processing the priorities so that the model produces a unique solution; and (b) computing non-unique solutions that result from different priorities and then post-processing these into one solution. In either case, the pre/postprocessing is mathematically formulated as a convex combination of priorities/flows. 
a. Pre-processing of the priorities. For every $i$ with multiple-valued priorities $\alpha_{i j}$ and $\alpha_{i k}$, a representative priority $\alpha_{i}$ is computed through:

with

$$
\alpha_{i}=\sum_{j} w_{i j} \alpha_{i j}+\sum_{k} w_{i k} \alpha_{i k} \quad \forall i
$$

$$
\begin{array}{ll}
w_{i j} \geq 0 \quad \& \quad w_{i k} \geq 0 & \forall i, j, k \\
\sum_{j} w_{i j}+\sum_{k} w_{i k}=1 & \forall i
\end{array}
$$

Different choices of the weights $w_{i j}$ and $w_{i k}$ define different pre-processing strategies:

1. If no further information is available, uniform weights can be chosen. Other averaging schemes are thinkable but require justification based on supplementary modeling assumptions. The model of Flötteröd \& Nagel (2005) - without internal supply constraints - resorts to this type of approach.

2. One representative $\alpha_{i j}$ (or $\alpha_{i k}$ ) could be selected by setting the respective $w_{i j}\left(w_{i k}\right)$ to one and all other weights to zero. This approach could be justified by the following considerations:

a. The (internal) supply constraint that follows most naturally from the history of flows could be selected. However, the analysis of Section 4 indicates that such a type of additional system memory can lead to undesirable results.

b. A ranking could be defined among all (internal) supply constraints so that the highest ranked active constraint determines the solution. This ranking could be made based on the amount of competition that is present for each supply. That way, the supply that is most 'overloaded' would determine the throughput. However, this competition or overloading cannot be directly determined from the demands $S_{i}$ as this would not be compliant with the invariance principle. Rather, this ranking should be based on the competition or overloading that arises in case this supply is indeed restrictive, i.e. considering capacities $C_{i}$ for supply constrained and $S_{i}$ for demand constrained links.

A clear advantage of the priority pre-processing approach is that it is relatively straightforward to implement, computationally efficient, and always guarantees a unique solution under any combination of boundary conditions. Furthermore, the resulting flow solution complies with all the requirements listed in Section 1.1 and all demand and (internal) supply constraints.

b. Post-processing of the flows. Every possible solution, resulting from the multiple, ambiguous priority ratios, is computed, leading to separate intersection flow patterns $\boldsymbol{q}_{r}$. These are then averaged into a unique resulting flow pattern $\boldsymbol{q}$ according to:

$$
\boldsymbol{q}=\sum_{r} w_{r} \boldsymbol{q}_{r} \quad \forall r
$$

with

$$
\begin{aligned}
& w_{r} \geq 0 \quad \forall r \\
& \sum_{r} w_{r}=1
\end{aligned}
$$


Again, different choices of the weights define different solution strategies:

1. One could assume a uniform average solution to hold. This is in line with the assumption that a deterministic DNL represents average network conditions. A naïve averaging, however, raises considerable difficulties. The average flow pattern may not comply with the requirement of individual flow maximization, or it may violate the invariance principle or some non-linear internal supply constraints.

2. One could also select a single solution as the most plausible or representative one for the given situation (this again corresponds to setting the respective weight $w_{r}$ to one and all others to zero). Again, this could be based on previous flows (assuming, e.g., that the smallest temporal change in flow patterns is the most plausible). Technically, this corresponds to uniquely selecting the priorities that have led to this solution, but the selection criterion is now based on flows, which are not known a priori in the priority pre-processing approach.

A drawback of the flow post-processing approach is that multiple candidate solutions need to be evaluated, rendering the intersection model computationally intensive. Also, as stated above, a true averaging of solutions can lead to flows that are inconsistent with the basic modeling assumptions.

Answering the question which of the above approaches or any other possible alternative is most realistic or most appropriate is difficult or even impossible without further theoretical and empirical research. Moreover, the answer might differ under varying circumstances. A trade-off between realism on the one hand and computational efficiency and limiting model complexity on the other seems inevitable.

\section{$4 \quad$ Spatial intersection modeling}

While the traditional point-like modeling approach is not disputed if internal supply constraints are disregarded, complex intersection models implement constraints that clearly have physical characteristics. Therefore, one might consider modeling intersections in DNL spatially, i.e. as mini-networks in which the conflict zones of crossing and merging flows are represented by dummy nodes, connected by dummy links. In this section, however, it is shown that spatial models exhibit similar as well as additional disadvantages within the macroscopic DNL framework.

Let us consider the example in Section 3.1.2 in a spatial model (Figure 9). Two dummy links connect two dummy nodes that represent the intersection conflict zones $k$. 


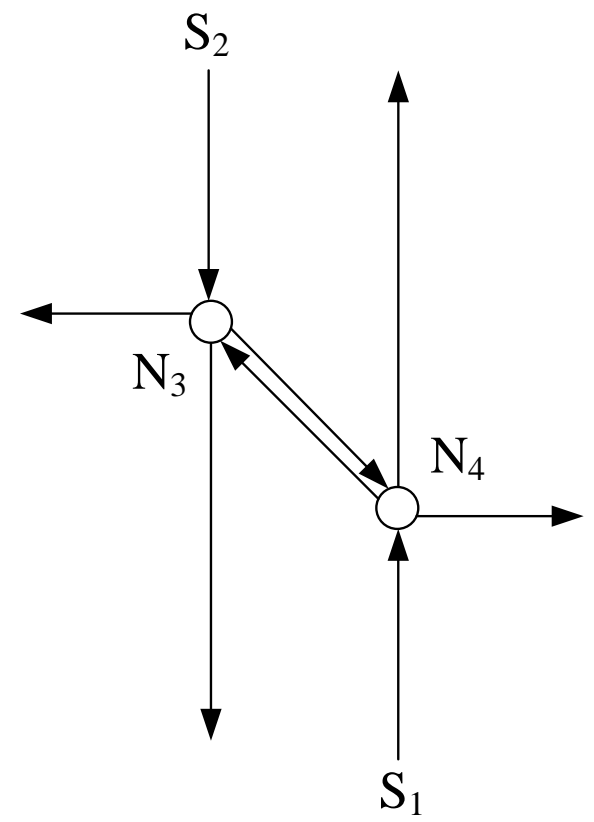

\section{Figure 9: Spatial Model of Example 2}

As in Section 3.1.2, the straight movements have absolute priority over the left movement of the other incoming link, and the left movements do not hinder each other. For simplicity's sake, the internal supplies are modeled identically to outgoing link supplies as a maximum number of vehicles that can cross the conflict zone per hour, with conflict zone capacities of $N_{k}=1000 \mathrm{veh} / \mathrm{h}:$

$$
N_{k}\left(q_{1}, q_{2}\right)=f_{1 k} q_{1}+f_{2 k} q_{2}-N_{k} \leq 0 \quad k=3,4
$$

Consequently, it is possible to simulate this spatial intersection model with any state-of-theart DNL model. Here, the Link Transmission Model (Yperman et al., 2007) is chosen, with a minor modification $^{2}$ to model the absolute compliance behavior in the internal conflicts.

Firstly, we consider two scenarios in which the boundary conditions external to the intersection are identical at the end of the simulation, but their histories are not (see Figure 10 and Figure 11).

\footnotetext{
${ }^{2}$ For this simple example, this requires nothing more than granting the prioritized movement its maximal share $\left(=S_{i k}\right)$ and passing the remaining internal supply $N_{k}-S_{i k}$ to the minor movement. Apart from that, this modification does not have any implication for the usual working of the DNL model.
} 

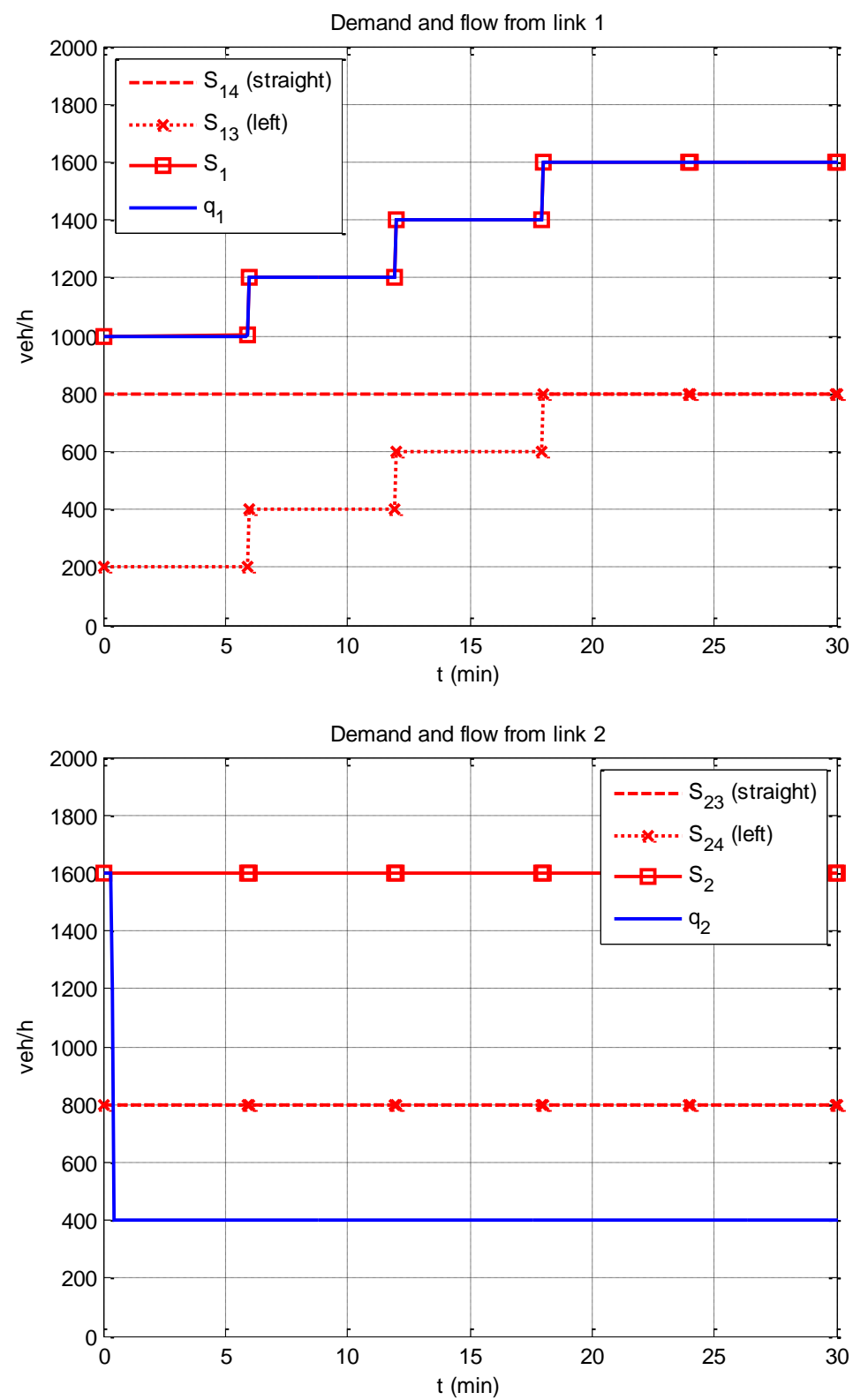

Figure 10: Scenario 1: $S_{13}$ increases gradually 

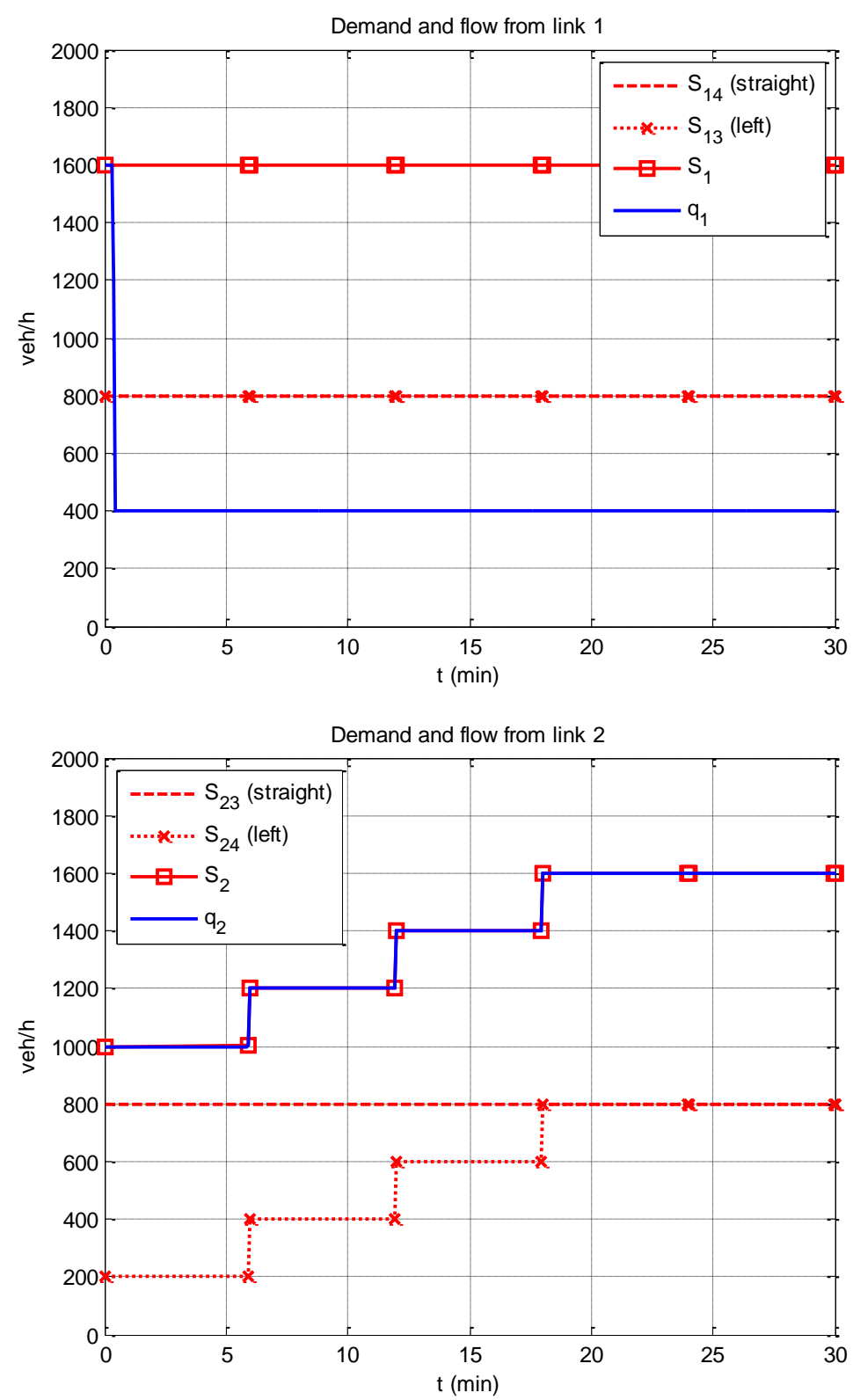

Figure 11: Scenario 1: $S_{24}$ increases gradually

In scenario 1 and 2, the left-turning demands are initially different. During the simulation, the low left-turning demand is gradually increased until it reaches the same level as the high leftturning demand from the opposing link.

In the initial phase of scenario $1, S_{13}$ is low, allowing link 1 to send its full demand, since there is enough remaining supply $\left(N_{3}-S_{23}=200 \mathrm{veh} / \mathrm{h}\right)$ for its minor left-turning movement. Meanwhile, the queue of the left-turning movement of link 2, which is obstructed by $N_{4}$, quickly spills back over the dummy link. This renders link 2 congested; also the straight movement is thus held back. While $S_{13}$ is gradually increased, this does not change the flows, as link 2 is now unable to claim its maximal share in $N_{3}$ (leaving $N_{3}-q_{23}=800 \mathrm{veh} / \mathrm{h}$ for link 1) due to the active constraint in $N_{4}$. In result, $q_{1}$ stays dominant throughout the simulation, while the other flow remains constrained. Scenario 2 is exactly the opposite.

Thus, although the boundary conditions at the end of the simulation are identical in both scenarios, the resulting flow patterns are very different. This demonstrates that also in a spatial model, solution uniqueness is not guaranteed given only the instantaneous boundary 
conditions. The spatial model inherently determines the solution on the basis of history, which is analogous to a point-like model that would solve the non-uniqueness based on history (see Section 3.4).

Now, one might state that determining the solution based on the history might not be an unrealistic option per se. However, in scenario 3, a history in which $S_{13}<S_{24}$ that lasts only a few seconds suffices for $q_{2}$ to take the advantage and hold it for the entire simulation. (The result is the opposite if $S_{13}>S_{24}$ holds initially.) Indeed, a time period equal to the spillback time over the left-turning dummy link is enough to block the straight flow coming from the same link. This means that during the initial phase of a DNL, starting from an empty network, a spatial intersection model could steer the simulation results solely based on which flows happen to reach an intersection first.
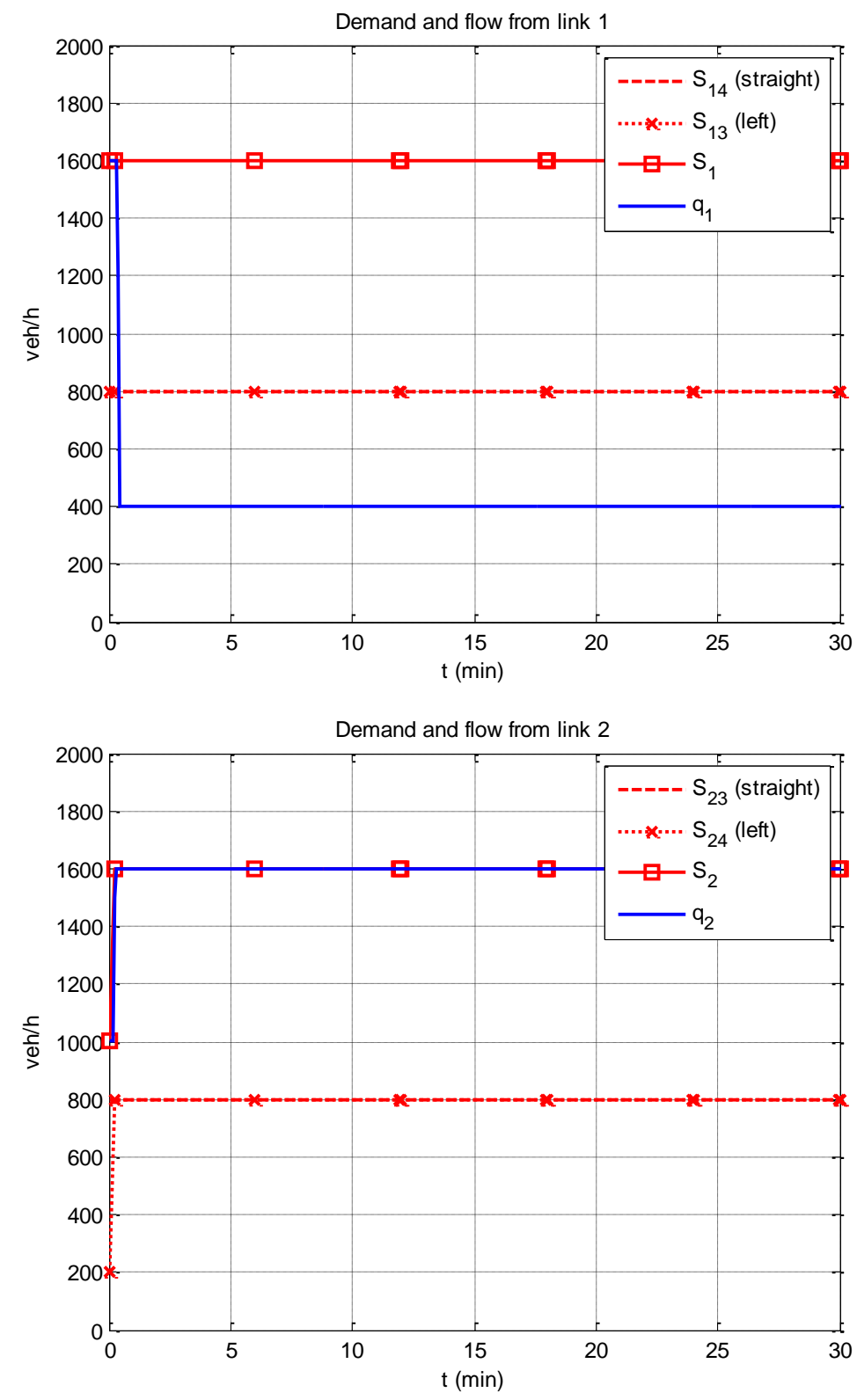

Figure 12: Scenario 3: A history of a few seconds fixes the solution 
Finally, a nearly symmetric demand pattern is chosen for scenario 4 . In result, an unstable flow pattern arises that converges slowly. In this converged solution, $q_{2}$ dominates $q_{1}$ thanks to a slightly smaller left turning fraction. The speed of convergence depends on the boundary conditions and the exact spatial representation (for instance the length of the dummy links and therewith the simulation update step).
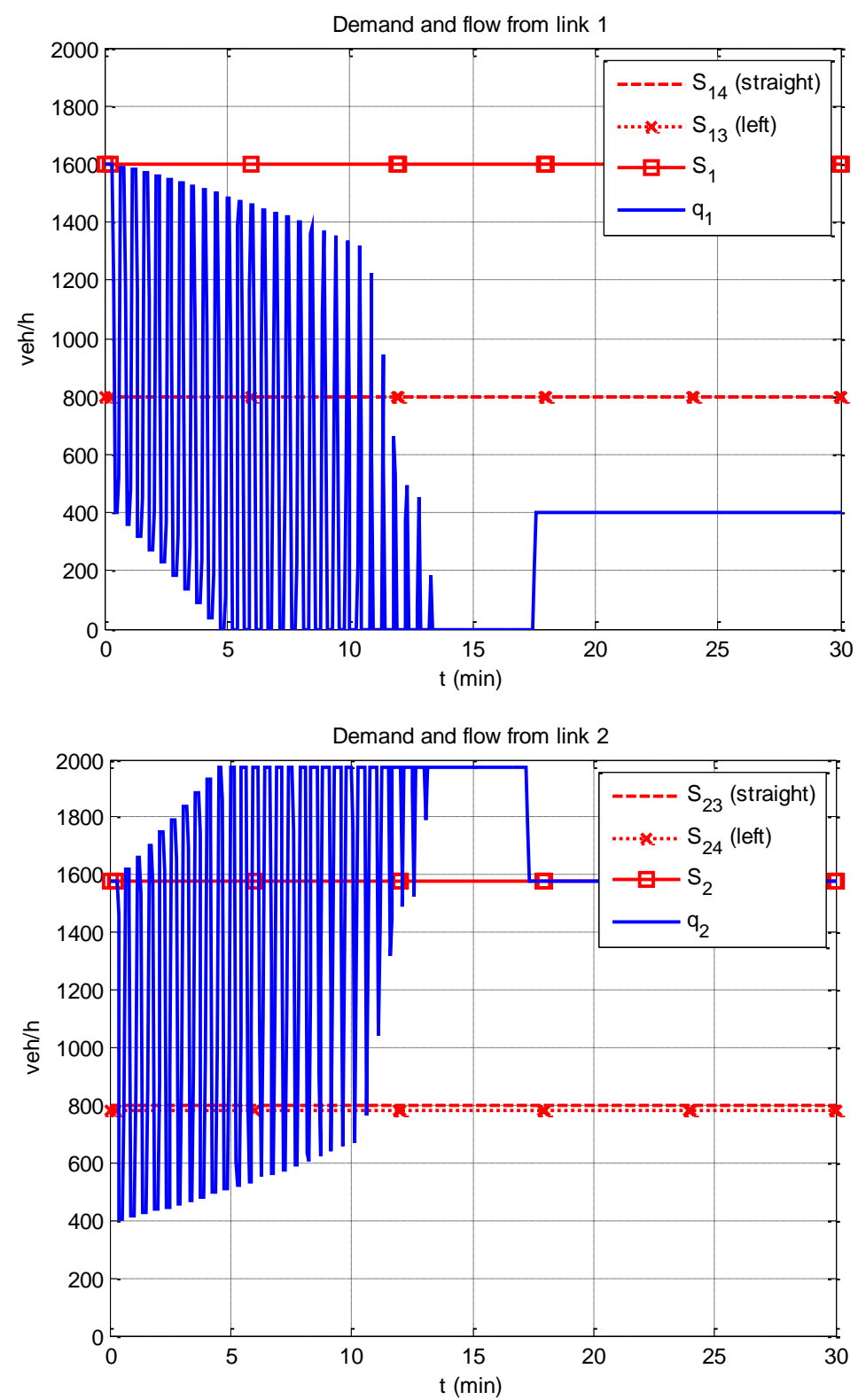

Figure 13: Scenario 4: Nearly symmetric demands engender unstable flows

The above illustrated behavior of spatial intersection models in a macroscopic DNL is clearly unrealistic and undesirable. While at first sight a spatial model physically detaches the mutual dependencies that lie at the origin of the ambiguity in point-like models, the wave propagation over the dummy links rejoins these dependencies. Mostly this rejoined ambiguity is immediately and irreversibly solved based on the history of flows, but sometimes, as in scenario 4, it causes an oscillating sequence of back and forth propagating waves on the dummy links. 
One flaw of this system lies in the application of macroscopic propagation theories designed for links of reasonable length on small dummy links that fit at most a few vehicles. A second problem that seems inherent to spatial intersection models is the spatial detachment of constraints that are subject to simultaneous driver decisions. Consider again the example in Figure 4. While in reality the two internal conflicts are evaluated simultaneously, they would be separated into two dummy nodes in the spatial model, leaving no option to capture the dependency between the two without further modeling efforts. Due to the before-mentioned problems, extreme caution is advised when inheriting networks for DNL or DTA purposes directly from static models, in which complex intersections are often modeled spatially.

While the possibility that a theory can be found that realistically treats propagation waves and simultaneous decisions in a spatial intersection model cannot be ruled out, it is safe to say that currently the problems and shortcomings of point-like intersection models cannot be bypassed in a spatial way. Simply regarding an intersection as a mini-network and trying to solve it in a standard macroscopic DNL manner produces unrealistic results. Partly, this behavior is similar to the non-uniqueness observed in point-like models, as different flow patterns can be obtained under instantaneously identical boundary conditions. Moreover, these flow patterns depend on the history of the boundary conditions and the exact spatial representation (including the dummy links' length and characteristics), which makes them difficult to control by the modeler.

As we feel that trying to include simultaneous decisions and accounting for mutually dependent flows naturally leads back to a point-like approach, we recommend that research on developing and improving traditional point-like intersection models is to be preferred. Two additional disadvantages of spatial models reinforce this argument:

- How to represent an intersection in a spatial model is a delicate matter. Caution is needed to make sure that the conflicts between the various movements are modeled realistically. For example, Chen et al. (2008) define their spatial intersection model as a grid of $2 \times 2$ cells, in which some movements that in reality would not be in conflict do hinder each other, whereas some other movements that are conflicting in reality are not in the model. Moreover, the configuration in Chen et al. (2008) can easily lead to model-induced, unrealistic gridlock. In Figure 14, an example of a gridlock-sensitive representation (a) and a non-gridlock-sensitive representation (b) of a standard $4 \times 4$ intersection is given. In essence, grouping movements into circular patterns may cause gridlock. 


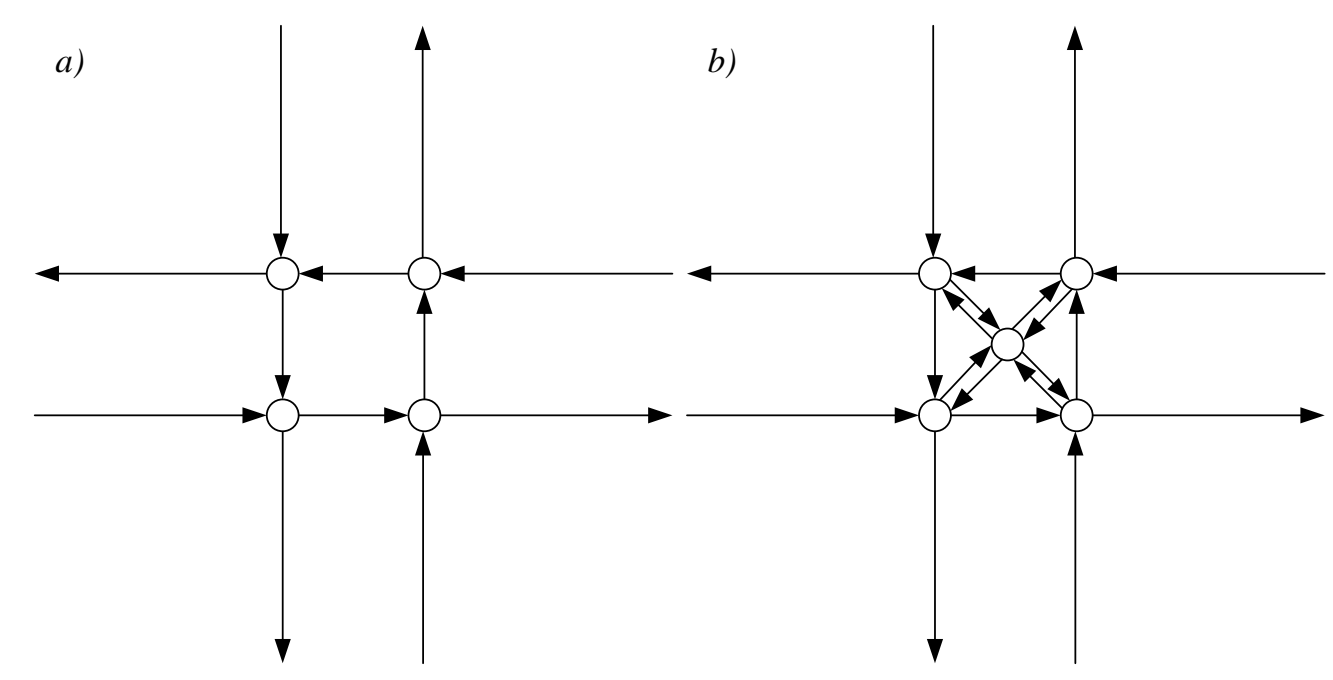

Figure 14: Gridlock-sensitive (a) and non-gridlock-sensitive (b) spatial intersection model

- The Courant-Friedrichs-Lewy condition - stating in the given context that the simulation time step must be no larger than the link travel time - constitutes a practical difficulty for the small dummy links of a spatial intersection model in that it increases the overall computation time in a network tremendously.

\section{$5 \quad$ Conclusion and outlook}

This paper discusses complex intersection modeling in macroscopic, first-order DNL. Building on previous research in Tampère et al. (2011) and Flötteröd \& Rohde (forthcoming), its main contributions are:

- the general definition of (internal) supply distribution through priority parameters, which are used to model different driver behavior

- the observation and analysis of solution non-uniqueness within this framework, which is found to result from realistic behavioral assumptions

- the formulation of a necessary and sufficient condition for solution uniqueness, which is however unlikely to be met on a complex intersection including internal conflicts

- the description of pragmatic approaches to remedy the non-uniqueness in deterministic point-like models

- the elaboration on the spatial modeling approach, revealing that this exhibits behavior that resembles the solution non-uniqueness of point-like models and furthermore shows unrealistic results in some cases

In this paper, many problems and concepts are discussed, but not always conclusively solved. To build realistic intersection models from these foundations does not only require to deal with their inherent non-uniqueness, but also to get further to the bottom of driver behavior in intersection conflicts.

Regarding the modeling of driver behavior in DNL, several open issues remain. A classification of different types of driver behavior is suggested, but further empirical validation on general (non-freeway) intersections is needed. In particular, specifications are to be developed for the internal intersection supply constraints that result from crossing and merging conflicts. Some theories exist that may serve as a basis, namely gap acceptance 
theory (Chapters 8 and 9 in Gartner et al., 2000) and conflict theory (Brilon \& Wu, 2001; Brilon \& Miltner, 2005). Hereby, we give preference to conflict theory since it seems more compatible with over-saturated conditions. Also, it is less complicated. Anyhow, further extension and adaptation to fit these theories into a DNL environment is required. The definition of the priority parameters that govern the internal supply distribution cannot be detached from this. Non-linear priority functions rather than constant priority ratios may be necessary to realistically capture the transition between different driver behavior, e.g. based on the saturation level. Also, caution is needed to preserve the model's compliance to the requirements listed in Section 1.1, following Tampère et al. (2011). Accounting for all of this should lead to a realistic, but also highly complex intersection model. Depending on the application, different trade-offs between realism, model complexity and data requirements will be desirable. Hopefully, this discussion will evolve comparably to that of link models, where various theories - e.g. travel time functions, vertical and spatial queuing - provide different levels of complexity and realism.

Regarding the treatment of the solution non-uniqueness, foremost empirical studies would be valuable that confirm the existence of multiple flow patterns under identical boundary conditions, and reveal their characteristics - e.g. probability, frequency of switches, duration of stable periods - and the (external) factors that lead to these characteristics - e.g. history, neighboring (signaled) intersections, the intersection geometry. This paper aids by helping to understand the phenomenon (and when it occurs) in the model, so that these specific circumstances can be sought for in the field. This empirical work - even if it disproves nonuniqueness in reality - can support the further research to develop and improve complex intersection models. Hereby, it seems that a point-like approach is most promising, upgrading the conceptual pragmatic approaches suggested in Section 3.4 - along with further developments of the behavioral modeling as described above - into unambiguous models for different types of intersections (AWSC, priority-controlled, signalized and roundabouts).

\section{References}

Adamo, V., Astarita, V., Florian, M., Mahut, M. \& Wu, J.H. (1999). Analytical modeling of intersections in traffic flow models with queue spill-back. IFORS $9915^{\text {Th }}$ Triennial Conference (ORSC), Beijing, P.R. China (also published in CRT di Montreal. Publication CRT 1999 nr. 52. National Libraries of Quebec and Canada).

Akcelik, R. \& Troutbeck R.J. (1991). Implementation of the Australian Roundabout Analysis Method in SIDRA. Highway Capacity and Level of Service, Proceedings of the International Symposium on Highway Capacity, pp. 17-34.

Bar-Gera, H. \& Ahn, S. (2010). Empirical macroscopic evaluation of freeway merge-ratios. Transportation Research Part C, 18 (4), pp.457-470.

Brilon, W. \& Wu, N. (2001). Capacity at unsignalized intersections derived by conflict technique. Transportation Research Record, Vol. 1776, pp. 82-90.

Brilon, W. \& Miltner, T. (2005). Capacity at intersections without traffic signals. Transportation Research Record, Vol. 1920, pp. 32-40. 
Buisson, C., Lesort, J.B. \& Lebacque, J.P. (1995). Macroscopic modeling of traffic flow and assignment in mixed networks. Proceedings of the Berlin ICCCBE Conference, pp. 13671374.

Carey, M. (1992). Nonconvexity of the Dynamic Traffic Assignment Problem. Transportation Research Part B, 26 (2), pp. 127-133.

Cassidy, M.J. \& Ahn, S. (2005). Driver Turn-Taking Behavior in Congested Freeway Merges. Transportation Research Record, Vol. 1934, pp. 140-147.

Chen, L., Jin, W-L. \& Zhang, J.H. (2008). An Urban Intersection Model Based on MultiCommodity Kinematic Wave Theories. Proceedings of the $11^{\text {th }}$ International IEEE Conference on ITS, pp. 269-274.

Chevallier, E. \& Leclercq, L. (2007). A macroscopic theory for unsignalized intersections. Transportation Research Part B, 41 (10), pp. 1139-1150.

Daganzo, C.F. (1995). The cell transmission model part II: Network traffic. Transportation Research Part B, 29 (2), pp. 79-93.

Daganzo, C.F. (1998). Queue spillovers in transportation networks with a route choice. Transportation Science, Vol. 32 (1), pp. 3-11.

Durlin, T. \& Henn, V. (2005). A Delayed Flow Intersection Model for Dynamic Traffic Assignment. Advanced OR and AI Methods in Transportation, Proceedings of the $10^{\text {th }}$ EWGT Meeting and $16^{\text {th }}$ Mini-EURO Conference, pp. 441-449.

Flötteröd, G. and Nagel, K. (2005). Some practical extensions to the cell transmission model. Proceedings of the 8th IEEE Intelligent Transportation Systems Conference, pp. 510-515.

Flötteröd, G. (2008). Traffic State Estimation with Multi-Agent Simulations. PhD dissertation, Berlin Institute of Technology, Germany.

Flötteröd, G., Bierlaire, M. \& Nagel, K. (forthcoming). Bayesian demand calibration for dynamic traffic simulations. Transportation Science.

Flötteröd, G. \& Rohde, J. (forthcoming). Modeling complex intersections with the celltransmission model. Transportation Research Part B.

Gartner, N.H., Messer, C.J. \& Rathi, A.K. (2000). Revised Monograph of Traffic Flow Theory, online publication of the Transportation Research Board, FHWA (http://www.tfhrc.gov/its/tft/tft.htm)

Gentile, G. (2010). The General Link Transmission Model for Dynamic Network Loading and a Comparison with the DUE Algorithm. New Developments in Transport Planning: Advances in Dynamic Traffic Assignment, pp. 153-178.

Gibb, J. (2011). A Model of Traffic Flow Constraint through Nodes for Dynamic Network Loading with Spillback. Presented at the $90^{\text {th }}$ Annual Meeting of the Transportation Research Board. 
Lebacque, J.P. \& Khoshyaran, M.M. (2005). First-order macroscopic traffic flow models: intersection modeling, network modeling. Proceedings of the $16^{\text {th }}$ International Symposium on Transportation and Traffic Theory (ISTTT), pp. 365-386.

Ngoduy, D., Hoogendoorn, S.P. \& van Lint, J.W.C. (2005). Modeling Traffic Flow Operation in Multilane and Multiclass Urban Networks. Transportation Research Record, Vol. 1923, pp.73-81.

Ni, D. \& Leonard, J.D. (2005). A simplified kinematic wave model at a merge bottleneck. Applied Mathematical Modelling, Vol. 29, pp. 1054-1072.

Ni, D., Leonard, J.D. \& Williams, B.M. (2006). The Network Kinematic Waves Model: A Simplified Approach to Network Traffic. Journal of Intelligent Transportation Systems 10 (1), pp. 1-14.

Osorio, C., Flötteröd, G. \& Bierlaire, M. (forthcoming). Dynamic network loading: a differentiable model that derives full link state distributions. Transportation Research Part B.

Raadsen, M.P.H., Mein, E.H., Schilpzand, M.P. \& Brandt, F. (2010). Implementation of a single dynamic traffic assignment model on mixed urban and highway transport networks including junction modelling. Proceedings of the $3^{\text {th }}$ DTA conference.

Sumalee, A., Zhong, R.X., Pan, T.L. \& Szeto, W.Y. (2011). Stochastic cell transmission model (SCTM): A stochastic dynamic traffic model for traffic state surveillance and assignment. Transportation Research Part B, 45 (3), pp. 507-533.

Taale, H. (2008). Integrated Anticipatory Control of Road Networks, A game-theoretical approach. T2008/15, TRAIL Thesis Series, the Netherlands.

Tampère, C.M.J., Corthout, R., D. Cattrysse \& L.H. Immers (2011), A generic class of first order node models for dynamic macroscopic simulation of traffic flows, Transportation Research Part B, 45 (1), pp. 289-309.

Troutbeck, R.J. \& Kako, S. (1999). Limited priority merge at unsignalized intersections. Transportation Research Part A, 33 (3-4), pp. 291-304.

Webster, F.V. (1958). Traffic Signal Settings. Road Research Laboratory Technical Paper No. 39.

Yperman, I., Tampère, C.M.J. \& Immers, L.H. (2007). A Kinematic Wave Dynamic Network Loading Model with Intersection Delays. Presented at the $86^{\text {th }}$ Annual Meeting of the Transportation Research Board. 


\section{Appendix: proof of (10) as a sufficient and necessary condition for uniqueness}

For this proof, the demand constraints are considered to be not restrictive. This does not constitute a loss of generality, since the demand constraints can never be a source of mutual dependency between flows $q_{i}$ and with that of non-uniqueness. In other words, restrictive demand constraints may alleviate some multiple solutions, but never create new ones. Supply constraints for which only one inflow competes are in that sense analogous to demand constraints and thus also not considered.

Also note that this proof is formulated for model definitions that meet the 7 requirements of Section 1.1 and the supply distribution rules described in Section 2.3 (and Tampère et al., 2011), in accordance with the rest of the paper.

For notational convenience, only constraints $j$ are represented in the remainder. However, any $j$ could be replaced with an internal supply constraint $k$, yielding an entirely analogous proof.

Consider the two possible ways in which inflows $q_{i}$ can be mutually dependent and end up in multiple solutions (see Section 3.2):

1. Two or more inflows $q_{i}$ are tied in a set of two or more supply constraints $j$.

2. Three or more inflows are mutually dependent in some "circular way".

First, option 1 is considered on a $2 \times 2$ example and option 2 on a $3 \times 3$ case. Afterwards, both are generalized to $I x J$.

\section{$\underline{2 \times 2 \text { case: }}$}

Consider two incoming links $i_{1}, i_{2}$ and two outgoing links $j_{1}$ and $j_{2}$. Assume multi-valued priority parameters $\alpha_{i j}$ and that two solutions A and B are possible from the two supply constraints. In solution A, the constraint in $j_{1}$ is binding, in B the constraint in $j_{2}$. Following (5)-(7):

$$
\begin{aligned}
& \mathrm{A}: \frac{q_{i_{1}}^{A}}{q_{i_{2}}^{A}}=\frac{\alpha_{i_{1_{1}} j_{1}}}{\alpha_{i_{2} j_{1}}} \\
& B: \frac{q_{i_{1}}^{B}}{q_{i_{2}}^{B}}=\frac{\alpha_{i_{1} j_{2}}}{\alpha_{i_{2} j_{2}}}
\end{aligned}
$$

If the model's solution is unique, the only possibility for both A and B to be feasible is if they are equal:

$$
q_{i_{1}}^{A}=q_{i_{1}}^{B} \text { and } q_{i_{2}}^{A}=q_{i_{2}}^{B}
$$

Combining (17) and (18) immediately leads to:

$$
\frac{\alpha_{i_{1} j_{1}}}{\alpha_{i_{2} j_{1}}}=\frac{\alpha_{i_{1} j_{2}}}{\alpha_{i_{2} j_{2}}}
$$

Obviously, if (10) holds then (19) becomes:

$$
\frac{\alpha_{i_{1}} \beta_{j_{1}}}{\alpha_{i_{2}} \beta_{j_{1}}}=\frac{\alpha_{i_{1}} \beta_{j_{2}}}{\alpha_{i_{2}} \beta_{j_{2}}} \Rightarrow(10) \text { is sufficient }
$$

(20) obviously holds and thus (10) is a sufficient condition for uniqueness in this $2 \times 2$ case. 
Now assume (10) does not hold, i.e. $\alpha_{i j} \neq \alpha_{i} \beta_{j}$ for exactly one $i j$ ( $n-1$ equalities can always be formed with $n$ positive variables). Assume for example $\alpha_{i_{2} j_{2}} \neq \alpha_{i_{2}} \beta_{j_{2}}$, and that yet (19) still holds:

$$
\frac{\alpha_{i_{1}} \beta_{j_{1}}}{\alpha_{i_{2}} \beta_{j_{1}}}=\frac{\alpha_{i_{1}} \beta_{j_{2}}}{\alpha_{i_{2} j_{2}}} \Leftrightarrow \alpha_{i_{2} j_{2}}=\alpha_{i_{2}} \beta_{j_{2}} \Rightarrow(10) \text { is necessary }
$$

Thus, if (10) does not hold then (19) cannot hold, hence the solution is non-unique. Thus (10) is also a necessary condition.

\section{3x3 case:}

Assume a case as in Figure 7, where no two incoming flows are tied in the same two outgoing constraints, but the mutual dependency exists in a circular manner (option 2). Following again (5)-(7), three solutions are possible that each bind two $q_{i}$ in the restrictive supply constraint:

$$
\begin{aligned}
& A: \frac{q_{i_{1}}^{A}}{q_{i_{2}}^{A}}=\frac{\alpha_{i_{1} j_{1}}}{\alpha_{i_{2} j_{1}}} \\
& B: \frac{q_{i_{2}}^{B}}{q_{i_{3}}^{B}}=\frac{\alpha_{i_{2} j_{2}}}{\alpha_{i_{3} j_{2}}} \\
& C: \frac{q_{i_{3}}^{C}}{q_{i_{1}}^{C}}=\frac{\alpha_{i_{3} j_{3}}}{\alpha_{i_{1} j_{3}}}
\end{aligned}
$$

Again, in case the model's solution is always unique, the only feasible possibility is:

$$
\begin{aligned}
& q_{i_{1}}^{A}=q_{i_{1}}^{C} \\
& q_{i_{2}}^{A}=q_{i_{2}}^{B} \\
& q_{i_{3}}^{B}=q_{i_{3}}^{C}
\end{aligned}
$$

Note that if two inflows are known and identical, the third is also known and identical under identical boundary conditions (since it is individually maximized). Thus, (23) suffices to describe the unique solution.

(22) and (23) yield:

(10) in (24) yields:

$$
\begin{aligned}
& \left(q_{i_{1}}^{A}=\right) \frac{\alpha_{i_{1} j_{1}}}{\alpha_{i_{2} j_{1}}} q_{i_{2}}^{A}=\frac{\alpha_{i_{1} j_{3}}}{\alpha_{i_{3} j_{3}}} q_{i_{3}}^{C} \text { and } q_{i_{3}}^{C}\left(=q_{i_{3}}^{B}\right)=\frac{\alpha_{i_{3} j_{2}}}{\alpha_{i_{2} j_{2}}} q_{i_{2}}^{A} \\
& \Rightarrow \frac{\alpha_{i_{1} j_{1}}}{\alpha_{i_{2} j_{1}}} q_{i_{2}}^{A}=\frac{\alpha_{i_{1} j_{3}}}{\alpha_{i_{3} j_{3}}} \frac{\alpha_{i_{3} j_{2}}}{\alpha_{i_{2} j_{2}}} q_{i_{2}}^{A} \Leftrightarrow \frac{\alpha_{i_{1} j_{1}}}{\alpha_{i_{2} j_{1}}} \frac{\alpha_{i_{2} j_{2}}}{\alpha_{i_{3} j_{2}}} \frac{\alpha_{i_{3} j_{3}}}{\alpha_{i_{1} j_{3}}}=1
\end{aligned}
$$

$$
\frac{\alpha_{i_{1}} \beta_{j_{1}}}{\alpha_{i_{2}} \beta_{j_{1}}} \frac{\alpha_{i_{2}} \beta_{j_{2}}}{\alpha_{i_{3}} \beta_{j_{2}}} \frac{\alpha_{i_{3}} \beta_{j_{3}}}{\alpha_{i_{1}} \beta_{j_{3}}}=1 \Rightarrow(10) \text { is sufficient }
$$

Assume again (10) does not hold, say $\alpha_{i_{1} j_{3}} \neq \alpha_{i_{1}} \beta_{j_{3}}$. If (24) were to still hold:

$$
\frac{\alpha_{i_{1}} \beta_{j_{1}}}{\alpha_{i_{2}} \beta_{j_{1}}} \frac{\alpha_{i_{2}} \beta_{j_{2}}}{\alpha_{i_{3}} \beta_{j_{2}}} \frac{\alpha_{i_{3}} \beta_{j_{3}}}{\alpha_{i_{1} j_{3}}}=1 \Leftrightarrow \alpha_{i_{1} j_{3}}=\alpha_{i_{1}} \beta_{j_{3}} \Rightarrow(10) \text { is necessary }
$$

\section{General IxJ case:}


Now, let us generalize the previous cases to a IxJ case. Naturally, many combinations of the mutual dependencies as described above may exist. For simplicity's sake, we will treat them separately.

Generalizing (17)-(19), there are a large number of possible solutions, which may be (nested) combinations of several supply constraints restricting several $q_{i}$. Still, isolating any combination of $i, i, j$ and $j^{\prime}$, the essence remains that the priority ratios of all mutually dependent flows should coincide in their mutual supply constraints:

(10) in (27) yields:

$$
\frac{\alpha_{i j}}{\alpha_{i^{\prime} j}}=\frac{\alpha_{i j^{\prime}}}{\alpha_{i^{\prime} j^{\prime}}} \quad \forall i, i^{\prime} \forall j, j^{\prime} \in F_{i} \cap F_{i^{\prime}}
$$

$$
\frac{\alpha_{i} \beta_{j}}{\alpha_{i^{\prime}} \beta_{j}}=\frac{\alpha_{i} \beta_{j^{\prime}}}{\alpha_{i^{\prime}} \beta_{j^{\prime}}} \quad \forall i, i^{\prime} \forall j, j^{\prime} \in F_{i} \cap F_{i^{\prime}} \Rightarrow(10) \text { is sufficient }
$$

Assume (10) does not hold, say $\alpha_{i^{\prime} j^{\prime}} \neq \alpha_{i^{\prime}} \beta_{j^{\prime}},(27)$ would imply:

$$
\frac{\alpha_{i} \beta_{j}}{\alpha_{i^{\prime}} \beta_{j}}=\frac{\alpha_{i} \beta_{j^{\prime}}}{\alpha_{i^{\prime} j^{\prime}}} \Leftrightarrow \alpha_{i^{\prime} j^{\prime}}=\alpha_{i^{\prime}} \beta_{j^{\prime}} \Rightarrow(10) \text { is necessary }
$$

Generalizing (22) to any circular dependency with $l$ members involved:

$$
\begin{aligned}
& A: \frac{q_{i_{1}}^{A}}{q_{i_{2}}^{A}}=\frac{\alpha_{i_{1} j_{1}}}{\alpha_{i_{2} j_{1}}} \\
& B: \frac{q_{i_{2}}^{B}}{q_{i_{3}}^{B}}=\frac{\alpha_{i_{2} j_{2}}}{\alpha_{i_{3} j_{2}}} \\
& \text {. } \\
& L: \frac{q_{i_{1}}^{L}}{q_{i_{1}}^{L}}=\frac{\alpha_{i_{l} j_{l}}}{\alpha_{i_{1} j_{l}}}
\end{aligned}
$$

Again, in case the model's solution is unique, the only feasible possibility is:

$$
\begin{aligned}
& q_{i_{1}}^{A}=q_{i_{1}}^{L} \\
& \cdot \\
& \cdot \\
& q_{i_{l}}^{L-1}=q_{i_{l}}^{L}
\end{aligned}
$$

Combining (30) and (31) leads to:

$$
\frac{\alpha_{i_{1} j_{1}}}{\alpha_{i_{2} j_{1}}} \frac{\alpha_{i_{2} j_{2}}}{\alpha_{i_{3} j_{2}}} \ldots \frac{\alpha_{i_{l} j_{l}}}{\alpha_{i_{1} j_{l}}}=1
$$

(10) in (32) yields:

$$
\frac{\alpha_{i_{1}} \beta_{j_{1}}}{\alpha_{i_{2}} \beta_{j_{1}}} \frac{\alpha_{i_{2}} \beta_{j_{2}}}{\alpha_{i_{3}} \beta_{j_{2}}} \ldots \frac{\alpha_{i_{l}} \beta_{j_{l}}}{\alpha_{i_{1}} \beta_{j_{l}}}=1 \Rightarrow(10) \text { is sufficient }
$$


Assume (10) does not hold, say $\alpha_{i_{1} j_{l}} \neq \alpha_{i_{1}} \beta_{j_{l}}$, while (32) holds:

$$
\frac{\alpha_{i_{1}} \beta_{j_{1}}}{\alpha_{i_{2}} \beta_{j_{1}}} \frac{\alpha_{i_{2}} \beta_{j_{2}}}{\alpha_{i_{3}} \beta_{j_{2}}} \ldots \frac{\alpha_{i_{l}} \beta_{j_{l}}}{\alpha_{i_{1} j_{l}}}=1 \Leftrightarrow \alpha_{i_{1} j_{l}}=\alpha_{i_{1}} \beta_{j_{l}} \Rightarrow(10) \text { is necessary }
$$

